\title{
Zied Garbouj
}

\section{Some generalizations of ascent and descent for linear operators}

Received: 19 February 2021 / Accepted: 1 June 2021 / Published online: 17 June 2021

(C) The Author(s) 2021

\begin{abstract}
The purpose of this paper is to present in linear spaces some results for new notions called $A$-left (resp., $A$-right) ascent and $A$-left (resp., $A$-right) descent of linear operators (where $A$ is a given operator) which generalize two important notions in operator theory: ascent and descent. Moreover, if $A$ is a positive operator, we obtain several properties of ascent and descent of an operator in semi-Hilbertian spaces. Some basic properties and many results related to the ascent and descent for a linear operator on a linear space Kaashoek (Math Ann 172:105-115, 1967), Taylor (Math Ann 163:18-49, 1966) are extended to these notions. Some stability results under perturbations by compact operators and operators having some finite rank power are also given for these notions.
\end{abstract}

Mathematics Subject Classification Primary 47A05; Secondary 47A55

\section{Introduction and terminologies}

The notions ascent and descent of a linear operator were introduced by Riesz [12] in connection with his investigation of compact linear operators. Heuser [7] considered these notions for a linear operator $T$ in a linear space $\mathrm{X}$ under the condition that $T$ is defined everywhere. The last condition was lifted by Taylor [18], whose treatment was completed by Kaashoek [8]. Kaashoek also provided a unified way of proving Taylor's results (see also [11,19]). Especially in recent years, various aspects of the problem related to generalization of these notions have appeared in the literature. Let us mention, for example, [14] for linear relations. In this paper, closely related to this problem, some new notions are considered and many properties are investigated. These new notions will be called $A$-left (resp., $A$-right) ascent and $A$-left (resp., $A$-right) descent, where $A$ is a given linear operator. Many results from $[8,9,18]$ are extended to these notions.

The class of operators with finite ascent and descent has been studied in a large number of papers. For example, the class of partial isometries on a complex Hilbert space with finite ascent and descent recently studied in $[1,13]$. The classes of partial isometries, quasi-isometries, contractions, and m-isometries in semiHilbertian spaces have been the object of some intensive study, especially by Arias et al. [2,3], Suciu [16,17], Sid Ahmed et al. [15] but also by Fongi et al. [5]. In this paper, if $A$ is a positive operator, we obtain several properties of ascent and descent of an operator in semi-Hilbertian spaces.

In this section, we set up notation and terminology and we introduce the notions of $A$-left (resp., $A$-right) ascent and $A$-left (resp., $A$-right) descent.

Z. Garbouj ( $\square)$

Institut Supérieur des Sciences Appliquées et de Technologie de Kairouan, Université de Kairouan, Kairouan, Tunisie

E-mail: zied.garbouj.fsm@gmail.com; zied.garbouj@issatkr.u-kairouan.tn

Z. Garbouj

Département de Mathématiques, Route périphérique Dar El Amen, 3100 Kairouan, Tunisie 
Throughout this paper, $\mathrm{X}$ will be a linear space over the field of complex numbers. We will denote by $\mathscr{L}(\mathrm{X})$ the set of all linear operators on $\mathrm{X}$. For an operator $T \in \mathscr{L}(\mathrm{X})$, write $\mathrm{N}(T)$ for its kernel and $\mathrm{R}(T)$ for its range. It is well known that

$$
\mathrm{N}\left(T^{n}\right) \subset \mathrm{N}\left(T^{n+1}\right) \text { and } \mathrm{R}\left(T^{n+1}\right) \subset \mathrm{R}\left(T^{n}\right),
$$

for all $n \in \mathbb{N}$, where $\mathbb{N}=\{0,1,2, \cdots\}$ denotes the set of all non-negative integers. The smallest non-negative integer for which there is equality is called the ascent of $T$ and the descent of $T$, denoted by $\operatorname{asc}(T)$ and des $(T)$, respectively. In case no such number exists the ascent or descent of $T$ is said to be infinite. The nullity and the defect of a linear operator $T$ are defined by $\alpha(T)=\operatorname{dim} \mathrm{N}(T), \beta(T)=\operatorname{dim} \mathrm{X} / \mathrm{R}(T)$.

The notations $\mathrm{N}_{A}^{\ell}(T)$ and $\mathrm{R}_{A}^{\ell}(T)$ for linear operators $A, T \in \mathscr{L}(\mathrm{X})$ denote the A-left kernel and the A-left range of $T$, defined by

$$
\mathrm{N}_{A}^{\ell}(T)=\mathrm{N}(A T), \quad \mathrm{R}_{A}^{\ell}(T)=\mathrm{R}(A T) .
$$

Furthermore, $\mathrm{N}_{A}^{r}(T)$ and $\mathrm{R}_{A}^{r}(T)$ denote the A-right kernel and the A-right range of $T$, defined by

$$
\mathrm{N}_{A}^{r}(T)=\mathrm{N}(T A), \quad \mathrm{R}_{A}^{r}(T)=\mathrm{R}(T A) .
$$

We shall denote by $\alpha_{A}^{\ell}(T)=\alpha(A T), \beta_{A}^{\ell}(T)=\beta(A T), \alpha_{A}^{r}(T)=\alpha(T A)$ and $\beta_{A}^{r}(T)=\beta(T A)$ its A-left nullity, A-left defect, $A$-right nullity, and $A$-right defect of $T$, respectively.

Remark 1.1 Let $\mathrm{H}$ be a Hilbert space with inner product $\langle\cdot, \cdot\rangle$. Given a positive bounded operator $A$ on $\mathrm{H}$, let $\langle\cdot, \cdot\rangle_{A}$ be the semi-inner product defined by

$$
\langle x, y\rangle_{A}:=\langle A x, y\rangle, \quad \forall x, y \in \mathrm{H} .
$$

By $\|\cdot\|_{A}$, we denote the semi-norm induced by $\langle\cdot, \cdot\rangle_{A}$, that is

$$
\|x\|_{A}:=\langle x, x\rangle_{A}^{1 / 2}=\left\|A^{1 / 2} x\right\|, \quad \forall x \in \mathrm{H} .
$$

$\left(\mathrm{H},\langle\cdot, \cdot\rangle_{A}\right)$ is called a semi-Hilbertian space (see [2,3]). We observe that if $T$ is a bounded operator on $\mathrm{H}$, and then

$$
\mathrm{N}_{A}^{\ell}(T)=\left\{x \in \mathrm{H}:\|T x\|_{A}=0\right\} .
$$

Indeed, by virtue of the fact that $\mathrm{N}(A)=\mathrm{N}\left(A^{1 / 2}\right)$, we get

$$
x \in \mathrm{N}_{A}^{\ell}(T) \Longleftrightarrow T x \in \mathrm{N}\left(A^{1 / 2}\right) \Longleftrightarrow\|T x\|_{A}=\left\|A^{1 / 2} T x\right\|=0 .
$$

We put

$$
\mathscr{L}_{\mathrm{M}}(\mathrm{X}):=\{T \in \mathscr{L}(\mathrm{X}): T(\mathrm{M}) \subset \mathrm{M}\},
$$

where $\mathrm{M}$ is a linear subspace of $\mathrm{X}$.

We present some useful properties concerning the $A$-left kernel, $A$-left range, $A$-right kernel, and $A$-right range of the sequence $\left(T^{n}\right)_{n \in \mathbb{N}} \subset \mathscr{L}(\mathrm{X})$.

Lemma 1.2 Let $A, T \in \mathscr{L}(\mathrm{X})$. Then

$$
\begin{aligned}
& \mathrm{R}_{A}^{\ell}\left(T^{n+m}\right) \subset \mathrm{R}_{A}^{\ell}\left(T^{n}\right), \quad \mathrm{N}_{A}^{r}\left(T^{n}\right) \subset \mathrm{N}_{A}^{r}\left(T^{n+m}\right), \quad \forall n, m \in \mathbb{N}, \\
& T \in \mathscr{L}_{\mathbf{R}(A)}(\mathbf{X}) \Longleftrightarrow \mathrm{R}_{A}^{r}\left(T^{n+m}\right) \subset \mathrm{R}_{A}^{r}\left(T^{n}\right), \quad \forall n, m \in \mathbb{N}, \\
& T \in \mathscr{L}_{\mathbf{N}(A)}(\mathbf{X}) \Longleftrightarrow \mathrm{N}_{A}^{\ell}\left(T^{n}\right) \subset \mathrm{N}_{A}^{\ell}\left(T^{n+m}\right), \quad \forall n, m \in \mathbb{N} .
\end{aligned}
$$

Proof The proof of this lemma is trivial.

Lemma 1.3 Let $A \in \mathscr{L}(\mathrm{X})$ and $T \in \mathscr{L}_{\mathrm{R}(A)}(\mathrm{X})$.

(1) If $\mathrm{N}_{A}^{r}\left(T^{k}\right)=\mathrm{N}_{A}^{r}\left(T^{k+1}\right)$ for some $k \in \mathbb{N}$, then $\mathrm{N}_{A}^{r}\left(T^{n}\right)=\mathrm{N}_{A}^{r}\left(T^{k}\right)$, for all non-negative integers $n \geq k$.

(2) If $\mathrm{R}_{A}^{r}\left(T^{k}\right)=\mathrm{R}_{A}^{r}\left(T^{k+1}\right)$ for some $k \in \mathbb{N}$, then $\mathrm{R}_{A}^{r}\left(T^{n}\right)=\mathrm{R}_{A}^{r}\left(T^{k}\right)$, for all non-negative integers $n \geq k$. 
Proof (1) Assume that $\mathrm{N}_{A}^{r}\left(T^{n+1}\right)=\mathrm{N}_{A}^{r}\left(T^{n}\right)$. It will be shown that $\mathrm{N}_{A}^{r}\left(T^{n+2}\right)=\mathrm{N}_{A}^{r}\left(T^{n+1}\right)$, and then, the statement will follow by induction. The inclusion $\mathrm{N}_{A}^{r}\left(T^{n+1}\right) \subset \mathrm{N}_{A}^{r}\left(T^{n+2}\right)$ is clear, so that only the converse inclusion remains to be proved. Let $x \in \mathrm{N}_{A}^{r}\left(T^{n+2}\right)$ and let $y=T A x \in \mathrm{R}(A)$, so that $y=A x_{0}$ for some $x_{0} \in \mathrm{X}$ and $T^{n+1} A x_{0}=T^{n+2} A x=0$. Thus, $x_{0} \in \mathrm{N}_{A}^{r}\left(T^{n+1}\right)=\mathrm{N}_{A}^{r}\left(T^{n}\right)$. Therefore, $T^{n+1} A x=T^{n} y=T^{n} A x_{0}=0$, so that $x \in \mathrm{N}_{A}^{r}\left(A^{n+1}\right)$, which implies (1).

(2) Assume that $\mathrm{R}_{A}^{r}\left(T^{n}\right)=\mathrm{R}_{A}^{r}\left(T^{n+1}\right)$. It suffices to show that $\mathrm{R}_{A}^{r}\left(T^{n+1}\right)=\mathrm{R}_{A}^{r}\left(T^{n+2}\right)$. Clearly, (1.2) shows that $\mathrm{R}_{A}^{r}\left(T^{n+2}\right) \subset \mathrm{R}_{A}^{r}\left(T^{n+1}\right)$. To see the converse inclusion, let $y \in \mathrm{R}_{A}^{r}\left(T^{n+1}\right)$, and then, $y=T^{n+1} A x$, for some $x \in \mathrm{X}$. Since $T^{n} A x \in \mathrm{R}_{A}^{r}\left(T^{n}\right)=\mathrm{R}_{A}^{r}\left(T^{n+1}\right)$, it follows that there exists $z \in \mathrm{X}$, such that $T^{n} A x=$ $T^{n+1} A z$, and hence, $y=T\left(T^{n} A x\right)=T^{n+2} A z \in \mathrm{R}_{A}^{r}\left(T^{n+2}\right)$. This leads to $\mathrm{R}_{A}^{r}\left(T^{n+1}\right) \subset \mathrm{R}_{A}^{r}\left(T^{n+2}\right)$ and the proof is complete.

Lemma 1.4 Let $A \in \mathscr{L}(\mathrm{X})$ and $T \in \mathscr{L}_{\mathrm{N}(A)}(\mathrm{X})$.

(1) If $\mathrm{N}_{A}^{\ell}\left(T^{k}\right)=\mathrm{N}_{A}^{\ell}\left(T^{k+1}\right)$ for some $k \in \mathbb{N}$, then $\mathrm{N}_{A}^{\ell}\left(T^{n}\right)=\mathrm{N}_{A}^{\ell}\left(T^{k}\right)$, for all non-negative integers $n \geq k$.

(2) If $\mathrm{R}_{A}^{\ell}\left(T^{k}\right)=\mathrm{R}_{A}^{\ell}\left(T^{k+1}\right)$ for some $k \in \mathbb{N}$, then $\mathrm{R}_{A}^{\ell}\left(T^{n}\right)=\mathrm{R}_{A}^{\ell}\left(T^{k}\right)$, for all non-negative integers $n \geq k$.

Proof (1) Assume that $\mathrm{N}_{A}^{\ell}\left(T^{n+1}\right)=\mathrm{N}_{A}^{\ell}\left(T^{n}\right)$. It will be shown that $\mathrm{N}_{A}^{\ell}\left(T^{n+2}\right)=\mathrm{N}_{A}^{\ell}\left(T^{n+1}\right)$, and then, the statement will follow by induction. From (1.3), we have $\mathrm{N}_{A}^{\ell}\left(T^{n+1}\right) \subset \mathrm{N}_{A}^{\ell}\left(T^{n+2}\right)$, so that only the converse inclusion remains to be proved. Let $x \in \mathrm{N}_{A}^{\ell}\left(T^{n+2}\right)$, and then, $T x \in \mathrm{N}_{A}^{\ell}\left(T^{n+1}\right)=\mathrm{N}_{A}^{\ell}\left(T^{n}\right)$. Thus, $x \in$ $\mathrm{N}_{A}^{\ell}\left(T^{n+1}\right)$, which implies (1).

(2) Assume that $\mathrm{R}_{A}^{\ell}\left(T^{n}\right)=\mathrm{R}_{A}^{\ell}\left(T^{n+1}\right)$. It suffices to show that $\mathrm{R}_{A}^{\ell}\left(T^{n+1}\right)=\mathrm{R}_{A}^{\ell}\left(T^{n+2}\right)$. First, it is shown that $\mathrm{R}_{A}^{\ell}\left(T^{n+1}\right) \subset \mathrm{R}_{A}^{\ell}\left(T^{n+2}\right)$. To see this let $y \in \mathrm{R}_{A}^{\ell}\left(T^{n+1}\right)$. Then, there exists $x \in \mathrm{X}$, such that $y=A T^{n+1} x$. Since

$$
\begin{aligned}
T^{n} x \in \mathrm{R}\left(T^{n}\right)+\mathrm{N}(A) & =A^{-1}\left(\mathrm{R}_{A}^{\ell}\left(T^{n}\right)\right) \\
& =A^{-1}\left(\mathrm{R}_{A}^{\ell}\left(T^{n+1}\right)\right)=\mathrm{R}\left(T^{n+1}\right)+\mathrm{N}(A),
\end{aligned}
$$

it follows that $T^{n} x=T^{n+1} z+w$, for some $z \in \mathbf{X}$ and $w \in \mathrm{N}(A)$. Therefore

$$
y=A T\left(T^{n} x\right)=A T^{n+2} z+A T w=A T^{n+2} z,
$$

because from (1.3), $\mathrm{N}(A) \subset \mathrm{N}_{A}^{\ell}(T)$. Hence $\mathrm{R}_{A}^{\ell}\left(T^{n+1}\right) \subset \mathrm{R}_{A}^{\ell}\left(T^{n+2}\right)$. Now, by (1.1), we conclude that $\mathrm{R}_{A}^{\ell}\left(T^{n+1}\right)=\mathrm{R}_{A}^{\ell}\left(T^{n+2}\right)$. This completes the proof.

For an operator $A \in \mathscr{L}(\mathrm{X})$, the statements in Lemmas 1.2 and 1.3 lead to the introduction of the $A$-right ascent and the $A$-right descent of $T \in \mathscr{L}_{\mathrm{R}(A)}(\mathrm{X})$ by

$$
\begin{aligned}
& \operatorname{asc}_{A}^{r}(T)=\inf \left\{k \in \mathbb{N}: \mathrm{N}_{A}^{r}\left(T^{k}\right)=\mathrm{N}_{A}^{r}\left(T^{k+1}\right)\right\}, \\
& \operatorname{des}_{A}^{r}(T)=\inf \left\{k \in \mathbb{N}: \mathrm{R}_{A}^{r}\left(T^{k}\right)=\mathrm{R}_{A}^{r}\left(T^{k+1}\right)\right\},
\end{aligned}
$$

respectively, whenever these minima exist. If no such numbers exist, the $A$-right ascent and $A$-right descent of $T$ are defined to be $\infty$. Likewise, the statements in Lemmas 1.2 and 1.4 lead to the introduction of the A-left ascent and the A-left descent of $T \in \mathscr{L}_{\mathrm{N}(A)}(\mathrm{X})$ by

$$
\begin{aligned}
& \operatorname{asc}_{A}^{\ell}(T)=\inf \left\{k \in \mathbb{N}: \mathbf{N}_{A}^{\ell}\left(T^{k}\right)=\mathbf{N}_{A}^{\ell}\left(T^{k+1}\right)\right\}, \\
& \operatorname{des}_{A}^{\ell}(T)=\inf \left\{k \in \mathbb{N}: \mathbf{R}_{A}^{\ell}\left(T^{k}\right)=\mathbf{R}_{A}^{\ell}\left(T^{k+1}\right)\right\},
\end{aligned}
$$

respectively, where the infimum over the empty set is taken to be infinite. The case when $A=I$, represents the ascent and descent of $T$, that is

$$
\operatorname{asc}_{A}^{\ell}(T)=\operatorname{asc}_{A}^{r}(T)=\operatorname{asc}(T), \operatorname{des}_{A}^{\ell}(T)=\operatorname{des}_{A}^{r}(T)=\operatorname{des}(T) .
$$

Remark 1.5 Suppose that $\mathrm{X}=\mathrm{H}$ is a Hilbert space, $T$ is a bounded operator on $\mathrm{H}$, and $A$ is a positive operator on $\mathrm{H}$.

(1) Recall that $T$ is an $A$-isometry (see [2, Definition 3.1]) if $\|T x\|_{A}=\|x\|_{A}$, for all $x \in \mathrm{H}$. We say that $T$ is an isometry if $T$ is an $I$-isometry. Obviously, if $T$ is an isometry, then $T$ is injective, i.e., $\operatorname{asc}(T)=0$. From [2, Proposition 3.2], we know also that if $T$ is an $A$-isometry, then $T(\mathrm{~N}(A)) \subset \mathrm{N}(A)$ and $\operatorname{asc}_{A}^{\ell}(T)=0$. 
(2) $T$ is said to be an $A$-quasi-isometry if $T^{*} A T=T^{* 2} A T^{2}$ (see [16]). If the relation is verified with $A=I$, $T$ is called a quasi-isometry. Suppose that $T$ is an $A$-quasi-isometry. Since

$$
\left\|T^{2} x\right\|_{A}=\|T x\|_{A}, \quad \forall x \in \mathrm{H},
$$

then if $T(\mathrm{~N}(A)) \subset \mathrm{N}(A)$, it follows by Remark 1.1 that $\operatorname{asc}_{A}^{\ell}(T) \leq 1$.

Remark 1.6 Let $A, T \in \mathscr{L}(\mathrm{X})$.

(1) Suppose that $T \in \mathscr{L}_{\mathrm{N}(A)}(\mathrm{X})$.

(a) $\operatorname{des}_{A}^{\ell}(T)=0$ if and only if $\mathrm{R}_{A}^{\ell}(T)=\mathrm{R}(A)$. In particular, $\operatorname{des}_{A}^{\ell}(T)=0$ when $\beta_{A}^{\ell}(T)=\beta(A)<+\infty$.

(b) $\operatorname{asc}_{A}^{\ell}(T)=0$ if and only if $\mathrm{N}_{A}^{\ell}(T)=\mathrm{N}(A)$. This shows that $\operatorname{asc}_{A}^{\ell}(T)=0$ if $\alpha_{A}^{\ell}(T)=\alpha(A)<+\infty$, since $\mathrm{N}(A) \subset \mathrm{N}_{A}^{\ell}(T)$.

(2) Suppose that $T \in \mathscr{L}_{\mathrm{R}(A)}(\mathrm{X})$.

(a) $\operatorname{des}_{A}^{r}(T)=0$ if and only if $\mathrm{R}_{A}^{r}(T)=\mathrm{R}(A)$. Since $\mathrm{R}_{A}^{r}(T) \subset \mathrm{R}(A)$, we infer that $\operatorname{des}_{A}^{r}(T)=0$ when $\beta_{A}^{r}(T)=\beta(A)<+\infty$.

(b) $\operatorname{asc}_{A}^{r}(T)=0$ if and only if $\mathrm{N}_{A}^{r}(T)=\mathrm{N}(A)$. This gives that $\operatorname{asc}_{A}^{r}(T)=0$ if $\alpha_{A}^{r}(T)=\alpha(A)<+\infty$.

(3) If $A T=T A$, then the subspaces $\mathrm{N}(A)$ and $\mathrm{R}(A)$ are invariant subspaces of $T$ and

$$
\operatorname{asc}_{A}^{\ell}(T)=\operatorname{asc}_{A}^{r}(T), \operatorname{des}_{A}^{\ell}(T)=\operatorname{des}_{A}^{r}(T) .
$$

(4) We note that if $\mathrm{R}\left(T^{n}\right)=\mathrm{R}\left(T^{n+1}\right)$, for some $n \in \mathbb{N}$, then $\mathrm{R}_{A}^{\ell}\left(T^{n}\right)=\mathrm{R}_{A}^{\ell}\left(T^{n+1}\right)$. This implies that

$$
\operatorname{des}_{A}^{\ell}(T) \leq \operatorname{des}(T), \quad \forall T \in \mathscr{L}_{\mathrm{N}(A)}(\mathrm{X}) .
$$

(5) If $T \in \mathscr{L}_{\mathrm{R}(A)}(\mathrm{X})$, then

$$
\operatorname{asc}_{A}^{r}(T) \leq \operatorname{asc}(T) .
$$

Indeed, assume that $\operatorname{asc}(T)=n<+\infty$ and $x \in \mathrm{N}_{A}^{r}\left(T^{n+1}\right)$, then $A x \in \mathrm{N}\left(T^{n+1}\right)=\mathrm{N}\left(T^{n}\right)$. Thus, $x \in \mathrm{N}_{A}^{r}\left(T^{n}\right)$ and by (1.1), we deduce that $\mathrm{N}_{A}^{r}\left(T^{n}\right)=\mathrm{N}_{A}^{r}\left(T^{n+1}\right)$. Hence, $\operatorname{asc}_{A}^{r}(T) \leq n$.

Next, we give examples of quantities introduced above.

Example 1.7 Let $L, S \in \mathscr{L}(\mathrm{X})$ and let $T \in \mathscr{L}(\mathrm{X} \times \mathrm{X})$ be the operator defined by

$$
T(x, y)=(S x, L y), \quad \forall(x, y) \in \mathbf{X} \times \mathbf{X} .
$$

The ascent and descent of $T$ are given by

$$
\operatorname{asc}(T)=\max \{\operatorname{asc}(S), \operatorname{asc}(L)\}, \operatorname{des}(T)=\max \{\operatorname{des}(S), \operatorname{des}(L)\} .
$$

Let $P \in \mathscr{L}(\mathrm{X})$ be a projection onto $\mathrm{N}(L)$ and $Q \in \mathscr{L}(\mathrm{X})$ be a projection with kernel $\mathrm{R}(L)$. Consider the operators $A$ and $B$ on $\mathrm{X} \times \mathrm{X}$ defined by

$$
A(x, y)=(x, P y), \quad B(x, y)=(x, Q y), \quad \forall x, y \in \mathbf{X} .
$$

Clearly, $\mathrm{R}(A)$ and $\mathrm{N}(B)$ are invariant subspaces of $T$ and

$$
T^{n} A(x, y)=\left(S^{n}(x), 0\right)=B T^{n}(x, y), \quad \forall x, y \in \mathbf{X},
$$

for all $n \in \mathbb{N} \backslash\{0\}$. This shows that

$$
\begin{aligned}
& \operatorname{asc}(S) \leq \operatorname{asc}_{A}^{r}(T) \leq \max \{1, \operatorname{asc}(S)\}, \quad \operatorname{des}(S) \leq \operatorname{des}_{A}^{r}(T) \leq \max \{1, \operatorname{des}(S)\}, \\
& \operatorname{asc}(S) \leq \operatorname{asc}_{B}^{\ell}(T) \leq \max \{1, \operatorname{asc}(S)\}, \quad \operatorname{des}(S) \leq \operatorname{des}_{B}^{\ell}(T) \leq \max \{1, \operatorname{des}(S)\} .
\end{aligned}
$$

We will distinguish four cases :

- Case 1 : If $\operatorname{asc}(L)=0$ (i.e., $\mathrm{N}(L)=\{0\}$ and $P=0$ ), then in (1.5), the equality $T^{n} A(x, y)=\left(S^{n}(x), 0\right)$ remains valid for $n=0$, and hence

$$
\operatorname{asc}_{A}^{r}(T)=\operatorname{asc}(S)=\operatorname{asc}(T), \operatorname{des}_{A}^{r}(T)=\operatorname{des}(S) \leq \operatorname{des}(T) .
$$


Since $\operatorname{asc}(L)=0$, then $\operatorname{des}(L)=0$ or $\operatorname{des}(L)=+\infty$. Therefore, the equality $\operatorname{des}_{A}^{r}(T)=\operatorname{des}(S)$ and the second equality in (1.4) imply that $\operatorname{des}_{A}^{r}(T)=\operatorname{des}(T)$ if $\operatorname{des}(L)=0$ or $\operatorname{des}(S)=+\infty$, and $\operatorname{des}_{A}^{r}(T)<$ $\operatorname{des}(T)=+\infty$ when $\operatorname{des}(S)<\operatorname{des}(L)=+\infty$.

- Case 2 : Suppose that $\operatorname{asc}(L)>0$, i.e., $\mathrm{N}(L) \backslash\{0\} \neq \emptyset$. For all $y \in \mathrm{N}(L) \backslash\{0\}$, we have $(0, y)=A(0, y) \in$ $\mathrm{R}(A)$ (resp., $\left.(0, y) \in \mathrm{N}_{A}^{r}(T)\right)$ but $(0, y) \notin \mathrm{R}_{A}^{r}(T)=\mathrm{R}(S) \times\{0\}$ (resp., $(0, y) \notin \mathrm{N}(A)$ ), this gives that $\operatorname{des}_{A}^{r}(T) \geq 1$ (resp., $\operatorname{asc}_{A}^{r}(T) \geq 1$ ). Now, by (1.6), it follows that:

$$
\operatorname{asc}_{A}^{r}(T)=\max \{1, \operatorname{asc}(S)\} \leq \operatorname{asc}(T), \operatorname{des}_{A}^{r}(T)=\max \{1, \operatorname{des}(S)\} .
$$

A combination of (1.4) and (1.7) leads to $\operatorname{asc}_{A}^{r}(T)=\operatorname{asc}(T)$ if $\operatorname{asc}(L)=1$ or $\operatorname{asc}(L) \leq \operatorname{asc}(S)$ and $\operatorname{asc}_{A}^{r}(T)<\operatorname{asc}(T)$ when $1 \leq \operatorname{asc}(S)<\operatorname{asc}(L)$. We also see that $\operatorname{asc}_{A}^{r}(T)<+\infty$ but $\operatorname{asc}(T)=+\infty$ when $\operatorname{asc}(S)<\operatorname{asc}(L)=+\infty$. Once by (1.4) and (1.7), we deduce that $\operatorname{des}_{A}^{r}(T)=\operatorname{des}(T)\left(\operatorname{resp} ., \operatorname{des}_{A}^{r}(T)<\right.$ $\left.\operatorname{des}(T), \operatorname{des}(T)<\operatorname{des}_{A}^{r}(T)\right)$ if $\operatorname{des}(L)=1$ or $\max \{1, \operatorname{des}(L)\} \leq \operatorname{des}(S)(\operatorname{resp} ., \max \{1, \operatorname{des}(S)\}<\operatorname{des}(L)$, $\operatorname{des}(L)=\operatorname{des}(S)=0)$. We also get $\operatorname{des}_{A}^{r}(T)<+\infty$, but $\operatorname{des}(T)=+\infty$ when $\operatorname{des}(S)<\operatorname{des}(L)=+\infty$.

We apply the foregoing reasoning to $\operatorname{asc}_{B}^{\ell}(T)$ and $\operatorname{des}_{B}^{\ell}(T)$, in the following two cases :

- Case 3 : If $\operatorname{des}(L)=0$ (in this case, $\operatorname{asc}(L)=0$ or $\operatorname{asc}(L)=+\infty$ ), then

$$
\operatorname{des}_{B}^{\ell}(T)=\operatorname{des}(S)=\operatorname{des}(T), \operatorname{asc}_{B}^{\ell}(T)=\operatorname{asc}(S) \leq \operatorname{asc}(T) .
$$

Therefore, by (1.4), we get $\operatorname{asc}_{B}^{\ell}(T)=\operatorname{asc}(T)$ if $\operatorname{asc}(L)=0$ or $\operatorname{asc}(S)=+\infty$ and $\operatorname{asc}_{B}^{\ell}(T)<\operatorname{asc}(T)=+\infty$ when $\operatorname{asc}(S)<\operatorname{asc}(L)=+\infty$.

- Case 4 : If $\operatorname{des}(L)>0$, then

$$
\operatorname{asc}_{B}^{\ell}(T)=\max \{1, \operatorname{asc}(S)\}, \quad \operatorname{des}_{B}^{\ell}(T)=\max \{1, \operatorname{des}(S)\} \leq \operatorname{des}(T) .
$$

From this and (1.4), we observe that $\operatorname{asc}_{B}^{\ell}(T)=\operatorname{asc}(T)\left(\operatorname{resp} ., \operatorname{asc}_{B}^{\ell}(T)<\operatorname{asc}(T), \operatorname{asc}(T)<\operatorname{asc}_{B}^{\ell}(T)\right)$ if $\operatorname{asc}(L)=1$ or $\max \{1, \operatorname{asc}(L)\} \leq \operatorname{asc}(S)$ (resp., $\max \{1, \operatorname{asc}(S)\}<\operatorname{asc}(L), \operatorname{asc}(L)=\operatorname{asc}(S)=0)$. We also observe that if $\operatorname{asc}(S)<\operatorname{asc}(L)=+\infty$, then $\operatorname{asc}_{B}^{\ell}(T)<+\infty$ but $\operatorname{asc}(T)=+\infty$.

For the relationships between the descent and the $B$-left descent of $T$, we have $\operatorname{des}_{B}^{\ell}(T)=\operatorname{des}(T)$ if $\operatorname{des}(L)=1$ or $\operatorname{des}(L) \leq \operatorname{des}(S)$ and $\operatorname{des}_{B}^{\ell}(T)<\operatorname{des}(T)$ when $1 \leq \operatorname{des}(S)<\operatorname{des}(L)$. We also have $\operatorname{des}_{B}^{\ell}(T)<+\infty$, but des $(T)=+\infty$ when $\operatorname{des}(S)<\operatorname{des}(L)=+\infty$.

Example 1.8 Let $\mathrm{H}$ be a separable Hilbert space with an orthonormal basis $\left(e_{i}\right)_{i \in \mathbb{N}}$.

(1) Define the linear operator $T$ in $\mathrm{H}$ by

$$
T\left(e_{i}\right)= \begin{cases}0 & \text { if } i=0 \\ e_{i-1} & \text { if } i \geq 1\end{cases}
$$

and let $A \in \mathscr{L}(\mathrm{H})$, such that $\mathrm{R}(A)$ is the subspace spanned by the vectors $\left(e_{i}\right)_{0 \leq i \leq k}$, for some $k \in \mathbb{N}$. Clearly, des $(T)=0, \operatorname{asc}(T)=+\infty$ and $T(\mathrm{R}(A)) \subset \mathrm{R}(A)$. Since

$$
\mathrm{N}\left(T^{k}\right) \varsubsetneqq \mathrm{N}\left(T^{k+1}\right)=\mathrm{R}(A),
$$

it follows that: $\operatorname{asc}_{A}^{r}(T)=\operatorname{des}_{A}^{r}(T)=k+1$.

(2) Let $T \in \mathscr{L}(\mathrm{H})$ be the operator defined by

$$
T\left(e_{i}\right)=e_{i+1}, \quad \forall i \in \mathbb{N} .
$$

Clearly, $\operatorname{asc}(T)=0$ and $\operatorname{des}(T)=+\infty$. Let $k \in \mathbb{N}$ and $A \in \mathscr{L}(\mathrm{H})$, such that $\mathrm{N}(A)$ is the subspace generated by $\left(e_{i}\right)_{i \geq k+1}$. It is not hard to see that $T(\mathrm{~N}(A)) \subset \mathrm{N}(A), A T^{k+1}\left(e_{i}\right)=0$, for all $i \in \mathbb{N}$ and $A T^{k}\left(e_{0}\right)=A\left(e_{k}\right) \neq 0$. Hence

$$
\operatorname{asc}_{A}^{\ell}(T)=\operatorname{des}_{A}^{\ell}(T)=k+1 .
$$

(3) Let $T$ be a $n$-left generalized partial isometry in $\mathrm{H}$ (see [6]), i.e., $T^{n} T^{*} T=T^{n}$ for some $n \in \mathbb{N}$, where $T^{*}$ is the adjoint of $T$. Let $A=T^{*} T$, we suppose that $T(\mathrm{R}(A)) \subset \mathrm{R}(A)$. First of all, we see that $T^{k} T^{*} T=T^{k}$, for all $k \geq n$, which shows that $\operatorname{asc}_{A}^{r}(T)<+\infty$ (resp., $\left.\operatorname{des}_{A}^{r}(T)<+\infty\right)$ if and only if $\operatorname{asc}(T)<+\infty$ (resp., $\operatorname{des}(T)<+\infty)$. We also see that

$$
\operatorname{des}_{A}^{r}(T) \leq \max \{n, \operatorname{des}(T)\},
$$

and by Remark 1.6, we have $\operatorname{asc}_{A}^{r}(T) \leq \operatorname{asc}(T)$. 
(4) Let $T$ be a $n$-right generalized partial isometry in $\mathrm{H}$ (see [6]), i.e., $T T^{*} T^{n}=T^{n}$ for some $n \in \mathbb{N}$. Let $A=T T^{*}$, and assume that $T(\mathrm{~N}(A)) \subset \mathrm{N}(A)$. First, from Remark 1.6, we have $\operatorname{des}_{A}^{\ell}(T) \leq \operatorname{des}(T)$. Since $A T^{i}=T^{i}$, for all $i \geq n$, it follows that $\operatorname{asc}_{A}^{\ell}(T)=+\infty$ (resp., $\left.\operatorname{des}_{A}^{\ell}(T)=+\infty\right)$ if and only if $\operatorname{asc}(T)=+\infty$ (resp., des $(T)=+\infty)$. We also deduce that

$$
\operatorname{asc}_{A}^{\ell}(T) \leq \max \{n, \operatorname{asc}(T)\} .
$$

The paper is organized as follows. In the next section, we first established some algebraic lemmas that will be used throughout this work. More precisely, the section presents isomorphism type results for the $A$-left (resp., $A$-right) kernel and the $A$-left (resp., $A$-right) range of a non-negative power of a linear operator (see $[8,18]$ for the case $A$ is injective or surjective). In Remark 2.4, we show that if $A$ an operator injective (resp., surjective), then $\operatorname{asc}_{A}^{\ell}(T)=\operatorname{asc}(T)$ and $\operatorname{des}_{A}^{\ell}(T)=\operatorname{des}(T)\left(\operatorname{resp} ., \operatorname{asc}_{A}^{r}(T)=\operatorname{asc}(T)\right.$ and $\left.\operatorname{des}_{A}^{r}(T)=\operatorname{des}(T)\right)$, for all linear operator $T$. In the third section, several results related to the ascent and descent for a linear operator on a linear space $[8,18]$ are extended to $A$-left (resp., $A$-right) ascent and descent. In Sect. 4, we are concerned with the stability of the $A$-left (resp., $A$-right) ascent and descent of an operator $T$, under perturbations by compact operators and operators having some finite rank power commuting with $T$. Finally, Sect. 5 is devoted to the study of relationship between the ascent and descent of an $A$-adjoint of an operator $T$ and $A$-left (resp., $A$-right) ascent and descent of $T$.

\section{Some preliminary lemmas}

The linear spaces $Y$ and $Z$ are said to be isomorphic whenever there exists a one-one linear mapping from $Y$ onto $Z$. For abbreviation, we use the symbol $Y \cong Z$ to denote that $Y$ and $Z$ are isomorphic. Let $M$ and $N$ be linear subspaces in the linear space $X$. If $M \cap N=\{0\}$, we write $M+N$ for $M+N$. If $N \subset M$, then $\frac{M}{N}$ (or $\mathrm{M} / \mathrm{N}$ ) denotes the quotient space $\mathrm{M}$ modulo $\mathrm{N}$.

In this section, we prove some algebraic results needed in this paper. We start this section with the following remark.

Remark 2.1 For all $T, S \in \mathscr{L}(\mathrm{X})$, we have

$$
\frac{\mathrm{N}(T S)}{\mathrm{N}(S)} \cong \mathrm{R}(S) \cap \mathrm{N}(T) \text { and } \frac{\mathrm{R}(T)}{\mathrm{R}(T S)} \cong \frac{\mathrm{X}}{\mathrm{R}(S)+\mathrm{N}(T)} .
$$

Indeed, let $\theta_{1}$ be the quotient map from $\mathrm{X}$ onto $\mathrm{R}(T) / \mathrm{R}(T S)$, sending $x \in \mathrm{X}$ to its class $\overline{T x}$, and let $\theta_{2}$ : $\mathrm{N}(T S) \longrightarrow \mathrm{R}(S) \cap \mathrm{N}(T)$ be the operator given by $\theta_{2}(x)=S x$, if $x \in \mathrm{N}(T S)$. One can easily check that $\theta_{1}$ and $\theta_{2}$ are surjective, $\mathrm{N}\left(\theta_{1}\right)=\mathrm{R}(S)+\mathrm{N}(T)$ and $\mathrm{N}\left(\theta_{2}\right)=\mathrm{N}(S)$. Which implies that $\theta_{1}$ (resp., $\theta_{2}$ ) induces an isomorphism between the spaces $\mathrm{R}(T) / \mathrm{R}(T S)$ and $\mathrm{X} /(\mathrm{R}(S)+\mathrm{N}(T)$ ) (resp., $\mathrm{N}(T S) / \mathrm{N}(S)$ and $\mathrm{R}(S) \cap \mathrm{N}(T)$ ).

Lemma 2.2 Let $A, T \in \mathscr{L}(\mathrm{X})$ and $n, k \in \mathbb{N}$. Then

$$
\frac{\mathrm{N}_{A}^{r}\left(T^{n+k}\right)}{\mathrm{N}_{A}^{r}\left(T^{n}\right)} \cong \mathrm{R}_{A}^{r}\left(T^{n}\right) \cap \mathrm{N}\left(T^{k}\right) .
$$

In addition, if $T(\mathrm{~N}(A)) \subset \mathrm{N}(A)$, we have

$$
\frac{\mathrm{N}_{A}^{\ell}\left(T^{n+k}\right)}{\mathrm{N}_{A}^{\ell}\left(T^{n}\right)} \cong \frac{\mathrm{N}_{A}^{\ell}\left(T^{k}\right) \cap \mathrm{R}\left(T^{n}\right)}{\mathrm{N}(A) \cap \mathrm{R}\left(T^{n}\right)} .
$$

Proof Let $n, k \in \mathbb{N}, S=T^{n} A$ and $L=T^{k}$, by Remark 2.1, we get

$$
\frac{\mathrm{N}_{A}^{r}\left(T^{n+k}\right)}{\mathrm{N}_{A}^{r}\left(T^{n}\right)}=\frac{\mathrm{N}(L S)}{\mathrm{N}(S)} \cong \mathrm{R}(S) \cap \mathrm{N}(L)=\mathrm{R}_{A}^{r}\left(T^{n}\right) \cap \mathrm{N}\left(T^{k}\right)
$$

Suppose that $T(\mathrm{~N}(A)) \subset \mathrm{N}(A)$, and then, $\mathrm{N}(A) \cap \mathrm{R}\left(T^{n}\right) \subset \mathrm{N}_{A}^{\ell}\left(T^{k}\right) \cap \mathrm{R}\left(T^{n}\right)$ (see (1.3)). Denote by $\bar{x}$ the equivalence class of $x$ relative to the quotient space

$$
\mathrm{Y}:=\frac{\mathrm{N}_{A}^{\ell}\left(T^{k}\right) \cap \mathrm{R}\left(T^{n}\right)}{\mathrm{N}(A) \cap \mathrm{R}\left(T^{n}\right)} .
$$


Hence, $\theta(x)=\overline{T^{n} x}$ defines a linear operator from $\mathrm{N}_{A}^{\ell}\left(T^{n+k}\right)$ to the quotient space $\mathrm{Y}$. It is easy to see that $\theta$ is surjective and $\mathrm{N}(\theta)=\mathrm{N}_{A}^{\ell}\left(T^{n}\right)$. This proves that

$$
\frac{\mathrm{N}_{A}^{\ell}\left(T^{n+k}\right)}{\mathrm{N}_{A}^{\ell}\left(T^{n}\right)} \cong \frac{\mathrm{N}_{A}^{\ell}\left(T^{k}\right) \cap \mathrm{R}\left(T^{n}\right)}{\mathrm{N}(A) \cap \mathrm{R}\left(T^{n}\right)} .
$$

The proof is therefore complete.

Lemma 2.3 Let $A, T \in \mathscr{L}(\mathrm{X})$. For all $n, k \in \mathbb{N}$, we have

$$
\frac{\mathrm{R}_{A}^{\ell}\left(T^{n}\right)}{\mathrm{R}_{A}^{\ell}\left(T^{n+k}\right)} \cong \frac{\mathrm{X}}{\mathrm{R}\left(T^{k}\right)+\mathrm{N}_{A}^{\ell}\left(T^{n}\right)}
$$

and

$$
\frac{\mathrm{R}_{A}^{r}\left(T^{n}\right)}{\mathrm{R}_{A}^{r}\left(T^{n+k}\right)} \cong \frac{\mathrm{R}(A)}{\mathrm{R}(A) \cap\left[\mathrm{R}_{A}^{r}\left(T^{k}\right)+\mathrm{N}\left(T^{n}\right)\right]} \text {, when } T(\mathrm{R}(A)) \subset \mathrm{R}(A) \text {. }
$$

Proof It follows from Remark 2.1 that:

$$
\frac{\mathrm{R}_{A}^{\ell}\left(T^{n}\right)}{\mathrm{R}_{A}^{\ell}\left(T^{n+k}\right)}=\frac{\mathrm{R}\left(A T^{n}\right)}{\mathrm{R}\left(A T^{n} T^{k}\right)} \cong \frac{\mathrm{X}}{\mathrm{R}\left(T^{k}\right)+\mathrm{N}\left(A T^{n}\right)}=\frac{\mathrm{X}}{\mathrm{R}\left(T^{k}\right)+\mathrm{N}_{A}^{\ell}\left(T^{n}\right)} .
$$

Assume that $T(\mathrm{R}(A)) \subset \mathrm{R}(A)$, by (1.2), we have $\mathrm{R}_{A}^{r}\left(T^{n+k}\right) \subset \mathrm{R}_{A}^{r}\left(T^{n}\right)$. Consider the linear operator $\theta$ defined by

$$
\begin{aligned}
\theta: \mathrm{R}(A) & \longrightarrow \mathrm{R}_{A}^{r}\left(T^{n}\right) / \mathrm{R}_{A}^{r}\left(T^{n+k}\right) \\
x & \longmapsto \overline{T^{n} x},
\end{aligned}
$$

where $\bar{x}$ denotes the equivalence class in the quotient space $\mathrm{R}_{A}^{r}\left(T^{n}\right) / \mathrm{R}_{A}^{r}\left(T^{n+k}\right)$ to which $x \in \mathrm{R}_{A}^{r}\left(T^{n}\right)$ belongs. Clearly, $\theta$ is surjective and $\mathrm{N}(\theta)=\mathrm{R}(A) \cap\left[\mathrm{R}_{A}^{r}\left(T^{k}\right)+\mathrm{N}\left(T^{n}\right)\right]$, this gives that

$$
\frac{\mathrm{R}_{A}^{r}\left(T^{n}\right)}{\mathrm{R}_{A}^{r}\left(T^{n+k}\right)} \cong \frac{\mathrm{R}(A)}{\mathrm{R}(A) \cap\left[\mathrm{R}_{A}^{r}\left(T^{k}\right)+\mathrm{N}\left(T^{n}\right)\right]} .
$$

The proof is complete.

Remark 2.4 Let $A, T \in \mathscr{L}(\mathrm{X})$.

(1) Suppose that $A$ is injective. For $n \in \mathbb{N}$, we have $\mathrm{N}_{A}^{\ell}\left(T^{n}\right)=\mathrm{N}\left(T^{n}\right)$ and

$$
\frac{\mathrm{R}_{A}^{\ell}\left(T^{n}\right)}{\mathrm{R}_{A}^{\ell}\left(T^{n+1}\right)} \cong \frac{\mathrm{X}}{\mathrm{R}(T)+\mathrm{N}_{A}^{\ell}\left(T^{n}\right)}=\frac{\mathrm{X}}{\mathrm{R}(T)+\mathrm{N}\left(T^{n}\right)} \cong \frac{\mathrm{R}\left(T^{n}\right)}{\mathrm{R}\left(T^{n+1}\right)}
$$

according to Remark 2.1 and Lemma 2.3. This leads to

$$
\operatorname{asc}_{A}^{\ell}(T)=\operatorname{asc}(T), \operatorname{des}_{A}^{\ell}(T)=\operatorname{des}(T) .
$$

(2) Suppose that $A$ is surjective. Let $n \in \mathbb{N}$, since $\mathrm{R}_{A}^{r}\left(T^{n}\right)=\mathrm{R}\left(T^{n}\right)$, it follows from Remark 2.1 and Lemma 2.2 that:

$$
\frac{\mathrm{N}_{A}^{r}\left(T^{n+1}\right)}{\mathrm{N}_{A}^{r}\left(T^{n}\right)} \cong \mathrm{R}_{A}^{r}\left(T^{n}\right) \cap \mathrm{N}(T)=\mathrm{R}\left(T^{n}\right) \cap \mathrm{N}(T) \cong \frac{\mathrm{N}\left(T^{n+1}\right)}{\mathrm{N}\left(T^{n}\right)}
$$

Which shows that

$$
\operatorname{asc}_{A}^{r}(T)=\operatorname{asc}(T), \operatorname{des}_{A}^{r}(T)=\operatorname{des}(T)
$$


Lemma 2.5 Let $A \in \mathscr{L}(\mathrm{X})$ and $T \in \mathscr{L}_{\mathrm{N}(A)}(\mathrm{X})$. For $i \in \mathbb{N}$, we have

$$
\frac{\mathrm{N}_{A}^{\ell}\left(T^{i+1}\right)}{\left(\mathrm{N}_{A}^{\ell}\left(T^{i}\right)+\mathrm{R}(T)\right) \cap \mathrm{N}_{A}^{\ell}\left(T^{i+1}\right)} \cong \frac{\mathrm{N}_{A}^{\ell}(T) \cap\left[\mathrm{R}\left(T^{i}\right)+\mathrm{N}(A)\right]}{\mathrm{N}_{A}^{\ell}(T) \cap\left[\mathrm{R}\left(T^{i+1}\right)+\mathrm{N}(A)\right]} .
$$

Proof Denote $\bar{x}$ the equivalence class of $x$ relative to the quotient space

$$
\mathrm{Y}:=\frac{\mathrm{N}_{A}^{\ell}(T) \cap\left[\mathrm{R}\left(T^{i}\right)+\mathrm{N}(A)\right]}{\mathrm{N}_{A}^{\ell}(T) \cap\left[\mathrm{R}\left(T^{i+1}\right)+\mathrm{N}(A)\right]} .
$$

Hence

$$
J(x)=\overline{T^{i} x}
$$

defines a linear operator form $\mathrm{N}_{A}^{\ell}\left(T^{i+1}\right)$ to the quotient space $\mathrm{Y}$. Next, it is shown that $J$ is surjective and

$$
\mathrm{N}(J)=\left(\mathrm{N}_{A}^{\ell}\left(T^{i}\right)+\mathrm{R}(T)\right) \cap \mathrm{N}_{A}^{\ell}\left(T^{i+1}\right),
$$

which implies that $J$ induces an isomorphism between the spaces $\mathrm{N}_{A}^{\ell}\left(T^{i+1}\right) / \mathrm{N}(J)$ and $\mathrm{Y}$.

Now, let us show that $\mathrm{N}(J)=\left(\mathrm{N}_{A}^{\ell}\left(T^{i}\right)+\mathrm{R}(T)\right) \cap \mathrm{N}_{A}^{\ell}\left(T^{i+1}\right)$. Assume that $x \in \mathrm{N}(J)$, so that $T^{i} x \in$ $\mathrm{N}_{A}^{\ell}(T) \cap\left[\mathrm{R}\left(T^{i+1}\right)+\mathrm{N}(A)\right]$, which implies that $x \in \mathrm{N}_{A}^{\ell}\left(T^{i+1}\right)$ and $T^{i} x=T^{i+1} z+y$ for some $z \in \mathrm{X}$ and $y \in \mathrm{N}(A)$. Therefore, $T^{i}(x-T z)=y \in \mathrm{N}(A)$; this gives that $x-T z \in \mathrm{N}_{A}^{\ell}\left(T^{i}\right)$. It follows that $x \in \mathrm{N}_{A}^{\ell}\left(T^{i}\right)+\mathrm{R}(T)$ and $\mathrm{N}(J) \subset\left(\mathrm{N}_{A}^{\ell}\left(T^{i}\right)+\mathrm{R}(T)\right) \cap \mathrm{N}_{A}^{\ell}\left(T^{i+1}\right)$. To show the converse inclusion, let $x \in\left(\mathrm{N}_{A}^{\ell}\left(T^{i}\right)+\mathrm{R}(T)\right) \cap \mathrm{N}_{A}^{\ell}\left(T^{i+1}\right)$, so that $x \in \mathrm{N}_{A}^{\ell}\left(T^{i+1}\right)$ and $x \in \mathrm{N}_{A}^{\ell}\left(T^{i}\right)+\mathrm{R}(T)$. Then, $T^{i} x \in \mathrm{N}_{A}^{\ell}(T)$ and $T^{i} x \in \mathrm{N}(A)+\mathrm{R}\left(T^{i+1}\right)$. Therefore, $\overline{T^{i} x}=0$ which shows that $x \in \mathrm{N}(J)$.

To show that $J$ is surjective, let $y \in \mathrm{N}_{A}^{\ell}(T) \cap\left[\mathrm{R}\left(T^{i}\right)+\mathrm{N}(A)\right]$. Since $\mathrm{N}(A) \subset \mathrm{N}_{A}^{\ell}(T)$, then

$$
\mathrm{N}_{A}^{\ell}(T) \cap\left[\mathrm{R}\left(T^{i}\right)+\mathrm{N}(A)\right]=\mathrm{N}_{A}^{\ell}(T) \cap \mathrm{R}\left(T^{i}\right)+\mathrm{N}(A) .
$$

This implies that $y=x+z$, for some $x \in \mathrm{N}_{A}^{\ell}(T) \cap \mathrm{R}\left(T^{i}\right)$ and $z \in \mathrm{N}(A)$. Therefore, $x=T^{i} x_{1}$, with $x_{1} \in \mathrm{N}_{A}^{\ell}\left(T^{i+1}\right)$. As $z \in \mathrm{N}(A) \subset \mathrm{N}_{A}^{\ell}(T) \cap\left[\mathrm{R}\left(T^{i+1}\right)+\mathrm{N}(A)\right]$, it follows that:

$$
J\left(x_{1}\right)=\overline{T^{i} x_{1}}=\bar{x}=\overline{x+z}=\bar{y} .
$$

Thus, $J$ is surjective, and the proof is complete.

Lemma 2.6 Let $A \in \mathscr{L}(\mathrm{X})$ and $T \in \mathscr{L}_{\mathrm{N}(A)}(\mathrm{X})$. For $i \in \mathbb{N}$, we have

$$
\operatorname{dim} \frac{\mathrm{N}_{A}^{\ell}(T)}{\mathrm{N}_{A}^{\ell}(T) \cap\left[\mathrm{R}\left(T^{i}\right)+\mathrm{N}(A)\right]}=\operatorname{dim} \frac{\mathrm{N}_{A}^{\ell}\left(T^{i}\right)}{\mathrm{N}_{A}^{\ell}\left(T^{i}\right) \cap[\mathrm{R}(T)+\mathrm{N}(A)]} .
$$

Proof First of all, we observe that it follows from [8, Lemma 2.2] and Lemma 2.5 that:

$$
\frac{\mathrm{N}_{A}^{\ell}(T) \cap\left[\mathrm{R}\left(T^{i}\right)+\mathrm{N}(A)\right]}{\mathrm{N}_{A}^{\ell}(T) \cap\left[\mathrm{R}\left(T^{i+1}\right)+\mathrm{N}(A)\right]} \cong \frac{\mathrm{N}_{A}^{\ell}\left(T^{i+1}\right)+\mathrm{R}(T)}{\mathrm{N}_{A}^{\ell}\left(T^{i}\right)+\mathrm{R}(T)},
$$

for all $i \in \mathbb{N}$. But then

$$
\begin{aligned}
\operatorname{dim} \frac{\mathrm{N}_{A}^{\ell}(T)}{\mathrm{N}_{A}^{\ell}(T) \cap\left[\mathrm{R}\left(T^{i}\right)+\mathrm{N}(A)\right]} & =\sum_{k=0}^{i-1} \operatorname{dim} \frac{\mathrm{N}_{A}^{\ell}(T) \cap\left[\mathrm{R}\left(T^{k}\right)+\mathrm{N}(A)\right]}{\mathrm{N}_{A}^{\ell}(T) \cap\left[\mathrm{R}\left(T^{k+1}\right)+\mathrm{N}(A)\right]} \\
& =\sum_{k=0}^{i-1} \operatorname{dim} \frac{\mathrm{N}_{A}^{\ell}\left(T^{k+1}\right)+\mathrm{R}(T)}{\mathrm{N}_{A}^{\ell}\left(T^{k}\right)+\mathrm{R}(T)}=\operatorname{dim} \frac{\mathrm{N}_{A}^{\ell}\left(T^{i}\right)+\mathrm{R}(T)}{\mathrm{N}(A)+\mathrm{R}(T)},
\end{aligned}
$$

and so, once again by [8, Lemma 2.2],

$$
\operatorname{dim} \frac{\mathrm{N}_{A}^{\ell}(T)}{\mathrm{N}_{A}^{\ell}(T) \cap\left[\mathrm{R}\left(T^{i}\right)+\mathrm{N}(A)\right]}=\operatorname{dim} \frac{\mathrm{N}_{A}^{\ell}\left(T^{i}\right)}{\mathrm{N}_{A}^{\ell}\left(T^{i}\right) \cap[\mathrm{R}(T)+\mathrm{N}(A)]} .
$$

The proof is complete. 
Lemma 2.7 Let $A \in \mathscr{L}(\mathrm{X})$ and $T \in \mathscr{L}_{\mathrm{R}(A)}(\mathrm{X})$. For $i \in \mathbb{N}$, we have

$$
\frac{\mathrm{N}\left(T^{i+1}\right) \cap \mathrm{R}(A)}{\left[\mathrm{N}\left(T^{i}\right)+\mathrm{R}_{A}^{r}(T)\right] \cap \mathrm{N}\left(T^{i+1}\right) \cap \mathrm{R}(A)} \cong \frac{\mathrm{N}(T) \cap \mathrm{R}_{A}^{r}\left(T^{i}\right)}{\mathrm{N}(T) \cap \mathrm{R}_{A}^{r}\left(T^{i+1}\right)} .
$$

Proof Let us first observe that if $x \in \mathrm{N}\left(T^{i+1}\right) \cap \mathrm{R}(A)$, then $T^{i} x \in \mathrm{N}(T) \cap \mathrm{R}_{A}^{r}\left(T^{i}\right)$. Therefore, $J(x)=\overline{T^{i} x}$ defines a linear operator from $\mathrm{N}\left(T^{i+1}\right) \cap \mathrm{R}(A)$ to the quotient space $\left(\mathrm{N}(T) \cap \mathrm{R}_{A}^{r}\left(T^{i}\right)\right) /\left(\mathrm{N}(T) \cap \mathrm{R}_{A}^{r}\left(T^{i+1}\right)\right)$. To show that $J$ is surjective and

$$
\mathrm{N}(J)=\left[\mathrm{R}\left(T^{i}\right)+\mathrm{R}_{A}^{r}(T)\right] \cap \mathrm{N}\left(T^{i+1}\right) \cap \mathrm{R}(A) .
$$

Let $y \in \mathrm{N}(T) \cap \mathrm{R}_{A}^{r}\left(T^{i}\right)$, and then, $y=T^{i} A z$, for some $z \in \mathrm{X}$. This implies that $A z \in \mathrm{N}\left(T^{i+1}\right) \cap \mathrm{R}(A)$ and $J(A z)=\overline{T^{i} A z}=\bar{y}$. Hence, $J$ maps onto $\left(\mathrm{N}(T) \cap \mathrm{R}_{A}^{r}\left(T^{i}\right)\right) /\left(\mathrm{N}(T) \cap \mathrm{R}_{A}^{r}\left(T^{i+1}\right)\right)$.

Let $x \in \mathrm{N}(J)$, so that $x \in \mathrm{N}\left(T^{i+1}\right) \cap \mathrm{R}(A)$ and $T^{i} x \in \mathrm{N}(T) \cap \mathrm{R}_{A}^{r}\left(T^{i+1}\right)$. Hence, $T^{i} x=T^{i+1} A y$, for some $y \in \mathrm{X}$, and therefore, $x-T A y \in \mathrm{N}\left(T^{i}\right)$. Which shows that $x \in\left[\mathrm{N}\left(T^{i}\right)+\mathrm{R}_{A}^{r}(T)\right] \cap \mathrm{N}\left(T^{i+1}\right) \cap \mathrm{R}(A)$. To show the converse inclusion, assume that $x \in\left[\mathrm{N}\left(T^{i}\right)+\mathrm{R}_{A}^{r}(T)\right] \cap \mathrm{N}\left(T^{i+1}\right) \cap \mathrm{R}(A)$, so that $x \in \mathrm{N}\left(T^{i+1}\right)$ and $x \in \mathrm{N}\left(T^{i}\right)+\mathrm{R}_{A}^{r}(T)$. Thus, $T^{i} x \in \mathrm{N}(T) \cap \mathrm{R}_{A}^{r}\left(T^{i+1}\right)$, this gives that $J(x)=\overline{T^{i} x}=0$. Consequently, $\mathrm{N}(J)=\left[\mathrm{R}\left(T^{i}\right)+\mathrm{R}_{A}^{r}(T)\right] \cap \mathrm{N}\left(T^{i+1}\right) \cap \mathrm{R}(A)$ and the mapping $J$ induces an isomorphism between the quotient spaces $\left(\mathrm{N}\left(T^{i+1}\right) \cap \mathrm{R}(A)\right) / \mathrm{N}(J)$ and $\mathrm{R}(J)$. The proof is complete.

Lemma 2.8 Let $A \in \mathscr{L}(\mathrm{X})$ and $T \in \mathscr{L}_{\mathrm{R}(A)}(\mathrm{X})$. For $i \in \mathbb{N}$, we have

$$
\operatorname{dim} \frac{\mathrm{N}(T) \cap \mathrm{R}(A)}{\mathrm{N}(T) \cap \mathrm{R}_{A}^{r}\left(T^{i}\right)}=\operatorname{dim} \frac{\mathrm{N}\left(T^{i}\right) \cap \mathrm{R}(A)}{\mathrm{N}\left(T^{i}\right) \cap \mathrm{R}_{A}^{r}(T)} .
$$

Proof First, note that $\left(\mathrm{N}\left(T^{i}\right)+\mathrm{R}_{A}^{r}(T)\right) \cap \mathrm{R}(A)=\mathrm{N}\left(T^{i}\right) \cap \mathrm{R}(A)+\mathrm{R}_{A}^{r}(T)$, for all $i \in \mathbb{N}$, because $\mathrm{R}_{A}^{r}(T) \subset$ $\mathrm{R}(A)$. Therefore

$$
\left[\mathrm{N}\left(T^{i}\right) \cap \mathrm{R}(A)+\mathrm{R}_{A}^{r}(T)\right] \cap \mathrm{R}(A)=\left(\mathrm{N}\left(T^{i}\right)+\mathrm{R}_{A}^{r}(T)\right) \cap \mathrm{R}(A),
$$

and so, by $[8$, Lemma 2.2], we obtain that

$$
\begin{aligned}
\frac{\mathrm{N}\left(T^{i+1}\right) \cap \mathrm{R}(A)}{\left[\mathrm{N}\left(T^{i}\right)+\mathrm{R}_{A}^{r}(T)\right] \cap \mathrm{N}\left(T^{i+1}\right) \cap \mathrm{R}(A)} & =\frac{\mathrm{N}\left(T^{i+1}\right) \cap \mathrm{R}(A)}{\left[\mathrm{N}\left(T^{i}\right) \cap \mathrm{R}(A)+\mathrm{R}_{A}^{r}(T)\right] \cap \mathrm{N}\left(T^{i+1}\right) \cap \mathrm{R}(A)} \\
& \cong \frac{\mathrm{N}\left(T^{i+1}\right) \cap \mathrm{R}(A)+\mathrm{R}_{A}^{r}(T)}{\mathrm{N}\left(T^{i}\right) \cap \mathrm{R}(A)+\mathrm{R}_{A}^{r}(T)} .
\end{aligned}
$$

Now, Lemma 2.7 shows that

$$
\frac{\mathrm{N}\left(T^{i+1}\right) \cap \mathrm{R}(A)+\mathrm{R}_{A}^{r}(T)}{\mathrm{N}\left(T^{i}\right) \cap \mathrm{R}(A)+\mathrm{R}_{A}^{r}(T)} \cong \frac{\mathrm{N}(T) \cap \mathrm{R}_{A}^{r}\left(T^{i}\right)}{\mathrm{N}(T) \cap \mathrm{R}_{A}^{r}\left(T^{i+1}\right)},
$$

for all $i \in \mathbb{N}$. Hence, for every $i \in \mathbb{N} \backslash\{0\}$

$$
\begin{aligned}
\operatorname{dim} \frac{\mathrm{N}(T) \cap \mathrm{R}(A)}{\mathrm{N}(T) \cap \mathrm{R}_{A}^{r}\left(T^{i}\right)} & =\sum_{k=0}^{i-1} \operatorname{dim} \frac{\mathrm{N}(T) \cap \mathrm{R}_{A}^{r}\left(T^{k}\right)}{\mathrm{N}(T) \cap \mathrm{R}_{A}^{r}\left(T^{k+1}\right)} \\
& =\sum_{k=0}^{i-1} \operatorname{dim} \frac{\mathrm{N}\left(T^{k+1}\right) \cap \mathrm{R}(A)+\mathrm{R}_{A}^{r}(T)}{\mathrm{N}\left(T^{k}\right) \cap \mathrm{R}(A)+\mathrm{R}_{A}^{r}(T)} \\
& =\operatorname{dim} \frac{\mathrm{N}\left(T^{i}\right) \cap \mathrm{R}(A)+\mathrm{R}_{A}^{r}(T)}{\mathrm{R}_{A}^{r}(T)} .
\end{aligned}
$$

Finally, by applying [8, Lemma 2.2], we deduce that

$$
\operatorname{dim} \frac{\mathrm{N}(T) \cap \mathrm{R}(A)}{\mathrm{N}(T) \cap \mathrm{R}_{A}^{r}\left(T^{i}\right)}=\operatorname{dim} \frac{\mathrm{N}\left(T^{i}\right) \cap \mathrm{R}(A)}{\mathrm{N}\left(T^{i}\right) \cap \mathrm{R}_{A}^{r}(T)},
$$

and the proof is complete. 
Lemma 2.9 Let $A, T \in \mathscr{L}(\mathrm{X})$. Then

$$
\beta_{A}^{\ell}\left(T^{k}\right)=\operatorname{dim} \frac{\mathrm{X}}{\mathrm{R}\left(T^{k}\right)+\mathrm{N}(A)}+\beta(A), \quad \forall k \in \mathbb{N} .
$$

Proof Let $k$ be a non-negative integer. From Remark 2.1, it follows that:

$$
\frac{\mathrm{R}(A)}{\mathrm{R}_{A}^{\ell}\left(T^{k}\right)} \cong \frac{\mathrm{X}}{\mathrm{R}\left(T^{k}\right)+\mathrm{N}(A)} .
$$

On the other hand, since $\mathrm{R}_{A}^{\ell}\left(T^{k}\right) \subset \mathrm{R}(A) \subset \mathrm{X}$, then

$$
\beta_{A}^{\ell}\left(T^{k}\right)=\operatorname{dim} \frac{\mathrm{X}}{\mathrm{R}\left(T^{k}\right)+\mathrm{N}(A)}+\beta(A) .
$$

The proof is complete.

The following result relates the $A$-right (resp., $A$-left) nullity and $A$-right (resp., $A$-left) defect of a linear operator to that of its powers.

Lemma 2.10 Let $A, T \in \mathscr{L}(\mathrm{X})$.

(1) If $T \in \mathscr{L}_{\mathrm{R}(A)}(\mathrm{X})$, for all $k \in \mathbb{N} \backslash\{0\}$, we have

$$
\alpha_{A}^{r}\left(T^{k}\right) \leq k \alpha_{A}^{r}(T), \quad \beta_{A}^{r}\left(T^{k}\right) \leq k \beta_{A}^{r}(T) .
$$

(2) If $T \in \mathscr{L}_{\mathrm{N}(A)}(\mathrm{X})$, for all $k \in \mathbb{N} \backslash\{0\}$, we have

$$
\alpha_{A}^{\ell}\left(T^{k}\right) \leq k \alpha_{A}^{\ell}(T), \quad \beta_{A}^{\ell}\left(T^{k}\right) \leq k \beta_{A}^{\ell}(T) .
$$

Proof (1) We proceed by induction; clearly, for $k=1, \alpha_{A}^{r}\left(T^{k}\right) \leq k \alpha_{A}^{r}(T)$. Suppose we have shown its validity for $1 \leq k \leq n$. Then, we can complete the proof by showing

$$
\alpha_{A}^{r}\left(T^{n+1}\right) \leq(n+1) \alpha_{A}^{r}(T) .
$$

We observe from (1.1) that $\mathrm{N}_{A}^{r}\left(T^{n+1}\right) \subset \mathrm{N}_{A}^{r}\left(T^{n}\right)$, so by Lemma 2.2 and (1.2), we see that

$$
\begin{aligned}
\alpha_{A}^{r}\left(T^{n+1}\right) & =\alpha_{A}^{r}\left(T^{n}\right)+\operatorname{dim} \mathrm{N}_{A}^{r}\left(T^{n+1}\right) / \mathrm{N}_{A}^{r}\left(T^{n}\right) \\
& =\alpha_{A}^{r}\left(T^{n}\right)+\operatorname{dim} \mathrm{R}_{A}^{r}\left(T^{n}\right) \cap \mathrm{N}(T) \\
& \leq n \alpha_{A}^{r}(T)+\operatorname{dim} \mathrm{R}(A) \cap \mathrm{N}(T) \\
& \leq n \alpha_{A}^{r}(T)+\operatorname{dim} \mathrm{N}_{A}^{r}(T) / \mathrm{N}(A) \\
& \leq n \alpha_{A}^{r}(T)+\alpha_{A}^{r}(T)=(n+1) \alpha_{A}^{r}(T) .
\end{aligned}
$$

To prove the second inequality in (1). Clearly, $\beta_{A}^{r}\left(T^{k}\right) \leq k \beta_{A}^{r}(T)$ is valid for $k=1$; suppose we have shown its validity for $1 \leq k \leq n$. Then, if we prove that $\beta_{A}^{r}\left(T^{n+1}\right) \leq(n+1) \beta_{A}^{r}(T)$, we can complete the proof. From (1.2), we get $\mathrm{R}_{A}^{r}\left(T^{n+1}\right) \subset \mathrm{R}_{A}^{r}\left(T^{n}\right) \subset \mathrm{X}$. But then, Lemma 2.3 and [8, Lemma 2.3] imply that

$$
\begin{aligned}
\beta_{A}^{r}\left(T^{n+1}\right) & =\beta_{A}^{r}\left(T^{n}\right)+\operatorname{dim} \frac{\mathrm{R}_{A}^{r}\left(T^{n}\right)}{\mathrm{R}_{A}^{r}\left(T^{n+1}\right)} \\
& =\beta_{A}^{r}\left(T^{n}\right)+\operatorname{dim} \frac{\mathrm{R}(A)}{\mathrm{R}(A) \cap\left(\mathrm{R}_{A}^{r}(T)+\mathrm{N}\left(T^{n}\right)\right)} \\
& \leq n \beta_{A}^{r}(T)+\operatorname{dim} \frac{\mathrm{X}}{\mathrm{R}_{A}^{r}(T)+\mathrm{N}\left(T^{n}\right)} \\
& \leq n \beta_{A}^{r}(T)+\beta_{A}^{r}(T)=(n+1) \beta_{A}^{r}(T) .
\end{aligned}
$$

(2) First, we prove by induction that $\alpha_{A}^{\ell}\left(T^{k}\right) \leq k \alpha_{A}^{\ell}(T)$, for all $k \geq 1$. The case $k=1$ is obvious. Suppose that $\alpha_{A}^{\ell}\left(T^{k}\right) \leq k \alpha_{A}^{\ell}(T)$. According to (1.3), $\mathrm{N}_{A}^{\ell}\left(T^{k}\right) \subset \mathrm{N}_{A}^{\ell}\left(T^{k+1}\right)$, which proves that

$$
\alpha_{A}^{\ell}\left(T^{k+1}\right)=\alpha_{A}^{\ell}\left(T^{k}\right)+\operatorname{dim} \frac{\mathbf{N}_{A}^{\ell}\left(T^{k+1}\right)}{\mathbf{N}_{A}^{\ell}\left(T^{k}\right)} .
$$


Then, we deduce from Lemma 2.2 that

$$
\begin{aligned}
\alpha_{A}^{\ell}\left(T^{k+1}\right) & \leq k \alpha_{A}^{\ell}(T)+\operatorname{dim} \frac{\mathrm{N}_{A}^{\ell}(T) \cap \mathrm{R}\left(T^{k}\right)}{\mathrm{N}(A) \cap \mathrm{R}\left(T^{k}\right)} \\
& \leq k \alpha_{A}^{\ell}(T)+\alpha_{A}^{\ell}(T)=(k+1) \alpha_{A}^{\ell}(T) .
\end{aligned}
$$

From this, it follows that $\alpha_{A}^{\ell}\left(T^{k}\right) \leq k \alpha_{A}^{\ell}(T)$, for all $k \geq 1$.

Now, let us show that $\beta_{A}^{\ell}\left(T^{k}\right) \leq k \beta_{A}^{\ell}(T)$, for all $k \geq 1$. Clearly, the inequality $\beta_{A}^{\ell}\left(T^{k}\right) \leq k \beta_{A}^{\ell}(T)$ is valid for $k=1$; suppose we have shown its validity for $1 \leq k \leq n$. From Lemma 1.2, we observe that $\mathrm{N}(A) \subset \mathrm{N}_{A}^{\ell}\left(T^{n}\right), \mathrm{R}_{A}^{\ell}\left(T^{n+1}\right) \subset \mathrm{R}_{A}^{\ell}\left(T^{n}\right) \subset \mathrm{X}$, and apply Lemmas 2.3 and 2.9 , and then

$$
\begin{aligned}
\beta_{A}^{\ell}\left(T^{n+1}\right)= & \beta_{A}^{\ell}\left(T^{n}\right)+\operatorname{dim} \frac{\mathrm{R}_{A}^{\ell}\left(T^{n}\right)}{\mathrm{R}_{A}^{\ell}\left(T^{n+1}\right)} \\
=\beta_{A}^{\ell}\left(T^{n}\right)+\operatorname{dim} \frac{\mathrm{X}}{\mathrm{R}(T)+\mathrm{N}_{A}^{\ell}\left(T^{n}\right)} & \leq n \beta_{A}^{\ell}(T)+\operatorname{dim} \frac{\mathrm{X}}{\mathrm{R}(T)+\mathrm{N}(A)} \\
& \leq n \beta_{A}^{\ell}(T)+\beta_{A}^{\ell}(T)=(n+1) \beta_{A}^{\ell}(T) .
\end{aligned}
$$

Hence, the inequality $\beta_{A}^{\ell}\left(T^{k}\right) \leq k \beta_{A}^{\ell}(T)$ is valid for $k=n+1$, and our proof is complete.

\section{$3 A$-left ascent, $A$-left descent, $A$-right ascent, and $A$-right descent}

Part of the results proved in this section improve and generalize some results of A. E. Taylor and M. A. Kaashoek $[8,18]$ concerning the relationship between ascent, descent, nullity, and defect of linear operators.

We first present some remarks.

Remark 3.1 Let $A \in \mathscr{L}(\mathrm{X})$, such that $\operatorname{dim} \mathrm{R}(A)=k<+\infty$. For all $T \in \mathscr{L}_{\mathrm{R}(A)}(\mathrm{X})$ and $S \in \mathscr{L}_{\mathrm{N}(A)}(\mathrm{X})$, we have

$$
\operatorname{asc}_{A}^{r}(T) \leq k, \operatorname{des}_{A}^{r}(T) \leq k, \operatorname{des}_{A}^{\ell}(S) \leq k .
$$

Indeed, assume that $\operatorname{des}_{A}^{r}(T) \geq k+1$, and then

$$
\mathrm{R}_{A}^{r}\left(T^{k+1}\right) \varsubsetneqq \mathrm{R}_{A}^{r}\left(T^{k}\right) \varsubsetneqq \cdots \varsubsetneqq \mathrm{R}_{A}^{r}(T) \varsubsetneqq \mathrm{R}(A) .
$$

This leads to $\operatorname{dim} \mathrm{R}(A) \geq k+1$, contrary to assumption. Thus, $\operatorname{des}_{A}^{r}(T) \leq k$. Now, assume that $\operatorname{asc}_{A}^{r}(T) \geq$ $k+1$, and then, by Remark 2.1, we have

$$
0<\operatorname{dim} \frac{\mathrm{N}_{A}^{r}(T)}{\mathrm{N}(A)}<\cdots<\operatorname{dim} \frac{\mathrm{N}_{A}^{r}\left(T^{k+1}\right)}{\mathrm{N}(A)}=\operatorname{dim} \mathrm{R}(A) \cap \mathrm{N}\left(T^{k+1}\right) \leq \operatorname{dim} \mathrm{R}(A) .
$$

It follows from this that $\operatorname{dim} \mathrm{R}(A) \geq k+1$, which contradicts the fact that $\operatorname{dim} \mathrm{R}(A)=k$. We must therefore conclude that $\operatorname{asc}_{A}^{r}(T) \leq k$. Finally, as in $(*)$, we can prove that $\operatorname{des}_{A}^{\ell}(S) \leq k$.

Remark 3.2 Let $A \in \mathscr{L}(\mathrm{X})$, such that $\operatorname{dim} \mathrm{X} / \mathrm{N}(A)=k<+\infty$. For all $T \in \mathscr{L}_{\mathrm{N}(A)}(\mathrm{X})$ and $S \in \mathscr{L}_{\mathrm{R}(A)}(\mathrm{X})$, we have

$$
\operatorname{asc}_{A}^{\ell}(T) \leq k, \operatorname{des}_{A}^{\ell}(T) \leq k, \operatorname{asc}_{A}^{r}(S) \leq k
$$

Indeed, assume that $\operatorname{asc}_{A}^{\ell}(T) \geq k+1$, and then, we obtain

$$
0<\operatorname{dim} \frac{\mathrm{N}_{A}^{\ell}(T)}{\mathrm{N}(A)}<\cdots<\operatorname{dim} \frac{\mathrm{N}_{A}^{\ell}\left(T^{k+1}\right)}{\mathrm{N}(A)} \leq \operatorname{dim} \frac{\mathrm{X}}{\mathrm{N}(A)},
$$


and hence, $\operatorname{dim} \mathrm{X} / \mathrm{N}(A) \geq k+1$. This contradiction shows that $\operatorname{asc}_{A}^{\ell}(T) \leq k$. Now, assume that $\operatorname{des}_{A}^{\ell}(T) \geq$ $k+1$, and then, by Remark 2.1 , we see that

$$
\begin{aligned}
0<\operatorname{dim} \frac{\mathrm{R}(A)}{\mathrm{R}_{A}^{\ell}(T)} & <\operatorname{dim} \frac{\mathrm{R}(A)}{\mathrm{R}_{A}^{\ell}\left(T^{2}\right)} \\
& \vdots \\
& <\operatorname{dim} \frac{\mathrm{R}(A)}{\mathrm{R}_{A}^{\ell}\left(T^{k}\right)}<\operatorname{dim} \frac{\mathrm{R}(A)}{\mathrm{R}_{A}^{\ell}\left(T^{k+1}\right)}=\operatorname{dim} \frac{\mathrm{X}}{\mathrm{R}\left(T^{k+1}\right)+\mathrm{N}(A)} \leq \operatorname{dim} \frac{\mathrm{X}}{\mathrm{N}(A)} .
\end{aligned}
$$

This gives that $\operatorname{dim} \mathrm{X} / \mathrm{N}(A) \geq k+1$, contrary to assumption. Thus, $\operatorname{des}_{A}^{\ell}(T) \leq k$. Finally, as in $(*)$, we can prove that $\operatorname{asc}_{A}^{r}(S) \leq k$.

Arguing as in [18, Lemmas 3.1 and 3.2], with Lemma 1.2, we get the following remark.

Remark 3.3 Let $A, T \in \mathscr{L}(\mathrm{X})$.

(1) Suppose that $T \in \mathscr{L}_{\mathrm{N}(A)}(\mathrm{X})$ and there exists $m \in \mathbb{N}$, such that $\alpha_{A}^{\ell}\left(T^{k}\right) \leq m$ (resp., $\beta_{A}^{\ell}\left(T^{k}\right) \leq m$ ) for all $k \in \mathbb{N}$, then $\operatorname{asc}_{A}^{\ell}(T) \leq m\left(\operatorname{resp}\right.$., $\left.\operatorname{des}_{A}^{\ell}(T) \leq m\right)$.

(2) Suppose that $T \in \mathscr{L}_{\mathrm{R}(A)}(\mathrm{X})$ and there exists $m \in \mathbb{N}$, such that $\alpha_{A}^{r}\left(T^{k}\right) \leq m$ (resp., $\beta_{A}^{r}\left(T^{k}\right) \leq m$ ) for all $k \in \mathbb{N}$, then $\operatorname{asc}_{A}^{r}(T) \leq m\left(\operatorname{resp}\right.$., $\left.\operatorname{des}_{A}^{r}(T) \leq m\right)$.

Let $p \in \mathbb{N}$ and $k \in \mathbb{N} \backslash\{0\}$. Recall from [11, Theorems V.6.3 and V.6.4] that for a linear operator $T \in \mathscr{L}(\mathrm{X})$, one has $\operatorname{asc}(T) \leq p(\operatorname{resp} ., \operatorname{des}(T) \leq p)$ if and only if $\mathrm{R}\left(T^{p}\right) \cap \mathrm{N}\left(T^{k}\right)=\{0\}\left(\operatorname{resp} ., \mathrm{R}\left(T^{k}\right)+\mathrm{N}\left(T^{p}\right)=\mathrm{X}\right)$. In the following, we will discuss these properties when $T$ has finite $A$-left ascent or $A$-left descent or $A$-right ascent or $A$-right descent.

Theorem 3.4 Let $A \in \mathscr{L}(\mathrm{X})$ and $T \in \mathscr{L}_{\mathrm{R}(A)}(\mathrm{X})$.

(1) If $\mathrm{N}(T) \cap \mathrm{R}_{A}^{r}\left(T^{p}\right)=\{0\}$ for some $p \in \mathbb{N}$, then $\operatorname{asc}_{A}^{r}(T) \leq p$.

(2) If asc $C_{A}^{r}(T) \leq p$ for some $p \in \mathbb{N}$, then $\mathrm{N}\left(T^{k}\right) \cap \mathrm{R}_{A}^{r}\left(T^{p}\right)=\{0\}$, for all $k \in \mathbb{N}$.

(3) If $\mathrm{R}(A) \subset \mathrm{R}_{A}^{r}(T)+\mathrm{N}\left(T^{p}\right)$ for some $p \in \mathbb{N}$, then $\operatorname{des}_{A}^{r}(T) \leq p$.

(4) If $\operatorname{des}_{A}^{r}(T) \leq p$ for some $p \in \mathbb{N}$, then $\mathrm{R}(A) \subset \mathrm{R}_{A}^{r}\left(T^{k}\right)+\mathrm{N}\left(T^{p}\right)$, for all $k \in \mathbb{N}$.

Proof (1)-(2) Let $p \in \mathbb{N}$ and $k \in \mathbb{N} \backslash\{0\}$. From Lemma 2.2, it follows that:

$$
\begin{aligned}
\operatorname{asc}_{A}^{r}(T) \leq p & \Longleftrightarrow \mathrm{N}_{A}^{r}\left(T^{p}\right)=\mathrm{N}_{A}^{r}\left(T^{p+k}\right) \\
& \Longleftrightarrow \mathrm{N}_{A}^{r}\left(T^{p+k}\right) / \mathrm{N}_{A}^{r}\left(T^{p}\right)=\{0\} \Longleftrightarrow \mathrm{N}\left(T^{k}\right) \cap \mathrm{R}_{A}^{r}\left(T^{p}\right)=\{0\} .
\end{aligned}
$$

(3)-(4) Let $p \in \mathbb{N}$ and $k \in \mathbb{N} \backslash\{0\}$. Lemma 2.3 shows that

$$
\begin{aligned}
\operatorname{des}_{A}^{r}(T) \leq p \Longleftrightarrow \mathrm{R}_{A}^{r}\left(T^{p}\right)=\mathrm{R}_{A}^{r}\left(T^{p+k}\right) & \Longleftrightarrow \mathrm{R}_{A}^{r}\left(T^{p}\right) / \mathrm{R}_{A}^{r}\left(T^{p+k}\right)=\{0\} \\
& \Longleftrightarrow \mathrm{R}(A)=\mathrm{R}(A) \cap\left(\mathrm{R}_{A}^{r}\left(T^{k}\right)+\mathrm{N}\left(T^{p}\right)\right) \\
& \Longleftrightarrow \mathrm{R}(A) \subset \mathrm{R}_{A}^{r}\left(T^{k}\right)+\mathrm{N}\left(T^{p}\right) .
\end{aligned}
$$

The proof is complete.

Theorem 3.5 Let $A \in \mathscr{L}(\mathrm{X})$ and $T \in \mathscr{L}_{\mathrm{N}(A)}(\mathrm{X})$.

(1) If $\mathrm{N}_{A}^{\ell}(T) \cap \mathrm{R}\left(T^{p}\right) \subset \mathrm{N}(A)$ for some $p \in \mathbb{N}$, then $\operatorname{asc}_{A}^{\ell}(T) \leq p$.

(2) If asc $c_{A}^{\ell}(T) \leq p$ for some $p \in \mathbb{N}$, then $\mathrm{N}_{A}^{\ell}\left(T^{k}\right) \cap \mathrm{R}\left(T^{p}\right) \subset \mathrm{N}(A)$, for all $k \in \mathbb{N}$.

(3) If $\mathrm{X}=\mathrm{R}(T)+\mathrm{N}_{A}^{\ell}\left(T^{p}\right)$ for some $p \in \mathbb{N}$, then $\operatorname{des}_{A}^{\ell}(T) \leq p$.

(4) If $\operatorname{des}_{A}^{\ell}(T) \leq p$ for some $p \in \mathbb{N}$, then $\mathrm{X}=\mathrm{R}\left(T^{k}\right)+\mathrm{N}_{A}^{\ell}\left(T^{p}\right)$, for all $k \in \mathbb{N}$.

Proof (1)-(2) Let $p \in \mathbb{N}$ and $k \in \mathbb{N} \backslash\{0\}$. Since $\mathrm{N}(A) \subset \mathbf{N}_{A}^{\ell}\left(T^{k}\right)$, from Lemma 2.2, we obtain

$$
\begin{aligned}
\operatorname{asc}_{A}^{\ell}(T) \leq p \Longleftrightarrow \mathrm{N}_{A}^{\ell}\left(T^{p}\right)=\mathrm{N}_{A}^{\ell}\left(T^{p+k}\right) & \Longleftrightarrow \mathrm{N}_{A}^{\ell}\left(T^{p+k}\right) / \mathrm{N}_{A}^{\ell}\left(T^{p}\right)=\{0\} \\
& \Longleftrightarrow \mathrm{N}_{A}^{\ell}\left(T^{k}\right) \cap \mathrm{R}\left(T^{p}\right)=\mathrm{N}(A) \cap \mathrm{R}\left(T^{p}\right) \\
& \Longleftrightarrow \mathrm{N}_{A}^{\ell}\left(T^{k}\right) \cap \mathrm{R}\left(T^{p}\right) \subset \mathrm{N}(A) .
\end{aligned}
$$

(3)-(4) Let $p \in \mathbb{N}$ and $k \in \mathbb{N} \backslash\{0\}$. Lemma 2.3 proves that

$$
\operatorname{des}_{A}^{\ell}(T) \leq p \Longleftrightarrow \mathrm{R}_{A}^{\ell}\left(T^{p}\right) / \mathrm{R}_{A}^{\ell}\left(T^{p+k}\right)=\{0\} \Longleftrightarrow \mathrm{X}=\mathrm{R}\left(T^{k}\right)+\mathrm{N}_{A}^{\ell}\left(T^{p}\right) .
$$

The proof is complete. 
In the rest of this section, the interrelations between $A$-left (resp., $A$-right) nullity and $A$-left (resp., $A$-right) defect, and $A$-left (resp., $A$-right) ascent and $A$-left (resp., $A$-right) descent are studied.

Theorem 3.6 Let $A, T \in \mathscr{L}(\mathrm{X})$.

(1) If $T \in \mathscr{L}_{\mathrm{R}(A)}(\mathrm{X})$ and if $\operatorname{asc}_{A}^{r}(T)$ and $\operatorname{des}_{A}^{r}(T)$ are finite, then $\operatorname{asc}_{A}^{r}(T)=\operatorname{des}_{A}^{r}(T)$.

(2) If $T \in \mathscr{L}_{\mathrm{N}(A)}(\mathrm{X})$ and if $\operatorname{asc}_{A}^{\ell}(T)$ and $\operatorname{des}_{A}^{\ell}(T)$ are finite, then $\operatorname{asc}_{A}^{\ell}(T)=\operatorname{des}_{A}^{\ell}(T)$.

Proof (1) Suppose that $p=\operatorname{asc}_{A}^{r}(T)>\operatorname{des}_{A}^{r}(T)=q$. Then, $\mathrm{R}_{A}^{r}\left(T^{p}\right)=\mathrm{R}_{A}^{r}\left(T^{q}\right)$, and hence, we have by Lemma 2.2

$$
\begin{aligned}
\operatorname{dim} \frac{\mathrm{N}_{A}^{r}\left(T^{q+1}\right)}{\mathrm{N}_{A}^{r}\left(T^{q}\right)} & =\operatorname{dim} \mathrm{R}_{A}^{r}\left(T^{q}\right) \cap \mathrm{N}(T) \\
& =\operatorname{dim} \mathrm{R}_{A}^{r}\left(T^{p}\right) \cap \mathrm{N}(T)=\operatorname{dim} \frac{\mathrm{N}_{A}^{r}\left(T^{p+1}\right)}{\mathrm{N}_{A}^{r}\left(T^{p}\right)}=0 .
\end{aligned}
$$

However, this implies $p=\operatorname{asc}_{A}^{r}(T) \leq q$, contradicting the assumption $p>q$. Hence, we must have $p \leq q$. It remains to prove that the case in which $p<q$ is impossible. From $p<q$, we infer that $\mathrm{N}_{A}^{r}\left(T^{q}\right)=\mathrm{N}_{A}^{r}\left(T^{p}\right)$. We need to show that

$$
\mathrm{R}(A) \cap \mathrm{N}\left(T^{p}\right)=\mathrm{R}(A) \cap \mathrm{N}\left(T^{q}\right) .
$$

Let $x \in \mathrm{R}(A) \cap \mathrm{N}\left(T^{q}\right)$, and then, $x=A\left(x_{0}\right)$, for some $x_{0} \in \mathrm{X}$. Therefore, $x_{0} \in \mathrm{N}_{A}^{r}\left(T^{q}\right)=\mathrm{N}_{A}^{r}\left(T^{p}\right)$ and hence $x=A\left(x_{0}\right) \in \mathrm{R}(A) \cap \mathrm{N}\left(T^{p}\right)$. Which shows that $\mathrm{R}(A) \cap \mathrm{N}\left(T^{q}\right)=\mathrm{R}(A) \cap \mathrm{N}\left(T^{p}\right)$, and as $\mathrm{R}_{A}^{r}(T) \subset \mathrm{R}(A)$, we obtain

$$
\begin{aligned}
\mathrm{R}(A) \cap\left(\mathrm{R}_{A}^{r}(T)+\mathrm{N}\left(T^{p}\right)\right) & =\mathrm{R}_{A}^{r}(T)+\mathrm{R}(A) \cap \mathrm{N}\left(T^{p}\right) \\
& =\mathrm{R}_{A}^{r}(T)+\mathrm{R}(A) \cap \mathrm{N}\left(T^{q}\right)=\mathrm{R}(A) \cap\left(\mathrm{R}_{A}^{r}(T)+\mathrm{N}\left(T^{q}\right)\right) .
\end{aligned}
$$

Thus, Lemma 2.3 proves that

$$
\begin{aligned}
\operatorname{dim} \frac{\mathrm{R}_{A}^{r}\left(T^{p}\right)}{\mathrm{R}_{A}^{r}\left(T^{p+1}\right)} & =\operatorname{dim} \frac{\mathrm{R}(A)}{\mathrm{R}(A) \cap\left(\mathrm{R}_{A}^{r}(T)+\mathrm{N}\left(T^{p}\right)\right)} \\
& =\operatorname{dim} \frac{\mathrm{R}_{(A)}}{\mathrm{R}(A) \cap\left(\mathrm{R}_{A}^{r}(T)+\mathrm{N}\left(T^{q}\right)\right)}=\operatorname{dim} \frac{\mathrm{R}_{A}^{r}\left(T^{q}\right)}{\mathrm{R}_{A}^{r}\left(T^{q+1}\right)}=0,
\end{aligned}
$$

so that $p \leq \operatorname{des}_{A}^{r}(T)=q$. This contradicts the fact that $p<q$. Hence, we must have $\operatorname{asc}_{A}^{r}(T)=\operatorname{des}_{A}^{r}(T)$.

(2) If $p=\operatorname{asc}_{A}^{\ell}(T)>\operatorname{des}_{A}^{\ell}(T)=q$, then by definition $\mathrm{R}_{A}^{\ell}\left(T^{p}\right)=\mathrm{R}_{A}^{\ell}\left(T^{q}\right)$ and in particular

$$
\mathrm{R}\left(T^{p}\right)+\mathrm{N}(A)=A^{-1}\left(\mathrm{R}_{A}^{\ell}\left(T^{p}\right)\right)=A^{-1}\left(\mathrm{R}_{A}^{\ell}\left(T^{q}\right)\right)=\mathrm{R}\left(T^{q}\right)+\mathrm{N}(A) .
$$

Since $\mathrm{N}(A) \subset \mathrm{N}_{A}^{\ell}(T)$, we get $\mathrm{R}\left(T^{p}\right) \cap \mathrm{N}_{A}^{\ell}(T)+\mathrm{N}(A)=\mathrm{R}\left(T^{q}\right) \cap \mathrm{N}_{A}^{\ell}(T)+\mathrm{N}(A)$. Now, by Lemma 2.2 and $[8$, Lemma 2.2], we deduce that

$$
\begin{aligned}
\operatorname{dim} \frac{\mathrm{N}_{A}^{\ell}\left(T^{q+1}\right)}{\mathrm{N}_{A}^{\ell}\left(T^{q}\right)} & =\operatorname{dim} \frac{\mathrm{N}_{A}^{\ell}(T) \cap \mathrm{R}\left(T^{q}\right)}{\mathrm{N}(A) \cap \mathrm{R}\left(T^{q}\right)} \\
& =\operatorname{dim} \frac{\mathrm{N}_{A}^{\ell}(T) \cap \mathrm{R}\left(T^{q}\right)+\mathrm{N}(A)}{\mathrm{N}(A)} \\
& =\operatorname{dim} \frac{\mathrm{N}_{A}^{\ell}(T) \cap \mathrm{R}\left(T^{p}\right)+\mathrm{N}(A)}{\mathrm{N}(A)}=\operatorname{dim} \frac{\mathrm{N}_{A}^{\ell}\left(T^{p+1}\right)}{\mathrm{N}_{A}^{\ell}\left(T^{p}\right)}=0,
\end{aligned}
$$

which implies that $p \leq q$. This contradiction shows that $p \leq q$. It remains to prove that the case in which $p<q$ is impossible. The inequality $p<q$ implies that $\mathrm{N}_{A}^{\ell}\left(T^{p}\right)=\mathrm{N}_{A}^{\ell}\left(T^{q}\right)$. Therefore, by Lemma 2.3, we obtain

$$
\begin{aligned}
\operatorname{dim} \frac{\mathrm{R}_{A}^{\ell}\left(T^{p}\right)}{\mathrm{R}_{A}^{\ell}\left(T^{p+1}\right)} & =\operatorname{dim} \frac{\mathrm{X}}{\mathrm{R}(T)+\mathrm{N}_{A}^{\ell}\left(T^{p}\right)} \\
& =\operatorname{dim} \frac{\mathrm{X}}{\mathrm{R}(T)+\mathrm{N}_{A}^{\ell}\left(T^{q}\right)}=\operatorname{dim} \frac{\mathrm{R}_{A}^{\ell}\left(T^{q}\right)}{\mathrm{R}_{A}^{\ell}\left(T^{q+1}\right)}=0 ;
\end{aligned}
$$

this gives that $q=\operatorname{des}_{A}^{\ell}(T) \leq p$, a contradiction. Hence, $p=q$, and this completes the proof. 
A combination of Theorems 3.4, 3.5, and 3.6 leads to the following result.

Corollary 3.7 Let $A, T \in \mathscr{L}(\mathrm{X})$.

(1) Suppose that $T \in \mathscr{L}_{\mathrm{R}(A)}(\mathrm{X})$.

(a) If there exists $k \in \mathbb{N} \backslash\{0\}$, such that

$$
\mathrm{R}(A)=\mathrm{R}_{A}^{r}\left(T^{k}\right)+\mathrm{N}\left(T^{k}\right) \cap \mathrm{R}(A),
$$

then $\operatorname{asc}_{A}^{r}(T)=\operatorname{des}_{A}^{r}(T) \leq k$.

(b) If $\operatorname{asc}_{A}^{r}(T)$ and $\operatorname{des}_{A}^{r}(T)$ are finite, then

$$
\mathrm{R}(A)=\mathrm{R}_{A}^{r}\left(T^{k}\right)+\mathrm{N}\left(T^{k}\right) \cap \mathrm{R}(A),
$$

where $k=\operatorname{asc}_{A}^{r}(T)=\operatorname{des}_{A}^{r}(T)$.

(2) Suppose that $T \in \mathscr{L}_{\mathrm{N}(A)}(\mathrm{X})$.

(a) If there exists $k \in \mathbb{N} \backslash\{0\}$, such that

$$
\mathrm{X}=\mathrm{R}\left(T^{k}\right)+\mathrm{N}_{A}^{\ell}\left(T^{k}\right) \text { and } \mathrm{R}\left(T^{k}\right) \cap \mathrm{N}_{A}^{\ell}\left(T^{k}\right) \subset \mathrm{N}(A),
$$

then $\operatorname{asc}_{A}^{\ell}(T)=\operatorname{des}_{A}^{\ell}(T) \leq k$.

(b) If $\operatorname{asc}_{A}^{\ell}(T)$ and $\operatorname{des}_{A}^{\ell}(T)$ are finite, then

$$
\begin{aligned}
& \qquad \mathrm{X}=\mathrm{R}\left(T^{k}\right)+\mathrm{N}_{A}^{\ell}\left(T^{k}\right) \text { and } \mathrm{R}\left(T^{k}\right) \cap \mathrm{N}_{A}^{\ell}\left(T^{k}\right) \subset \mathrm{N}(A), \\
& \text { where } k=\operatorname{asc}_{A}^{\ell}(T)=\operatorname{des}_{A}^{\ell}(T) \text {. }
\end{aligned}
$$

If either the $A$-left (resp., $A$-right) ascent or the $A$-left (resp., $A$-right) descent is finite, it is possible to obtain inequalities involving the $A$-left (resp., $A$-right) nullity and the $A$-left (resp., $A$-right) defect.

Theorem 3.8 Let $A \in \mathscr{L}(\mathrm{X})$ and $T \in \mathscr{L}_{\mathrm{N}(A)}(\mathrm{X})$. Suppose that $p=\operatorname{asc}_{A}^{\ell}(T)<+\infty$, and then

$$
\alpha_{A}^{\ell}(T)+\beta(A) \leq \beta_{A}^{\ell}(T)+\alpha(A),
$$

and there is equality in (3.1) if $\operatorname{des}_{A}^{\ell}(T)<+\infty$. Furthermore, if $\max \{\alpha(A), \beta(A)\}<+\infty, \min \left\{\alpha_{A}^{\ell}(T), \beta_{A}^{\ell}(T)\right\}$ $<+\infty$ and we have equality in (3.1), then $\operatorname{des}_{A}^{\ell}(T)<+\infty$.

Proof Since $p=\operatorname{asc}_{A}^{\ell}(T)<+\infty$, it follows from Theorem 3.5 that $\mathrm{N}_{A}^{\ell}(T) \cap \mathrm{R}\left(T^{p}\right) \subset \mathrm{N}(A)$. But then, [8, Lemma 2.2] and Lemma 2.6 show that

$$
\begin{aligned}
\operatorname{dim} \frac{\mathrm{N}_{A}^{\ell}(T)}{\mathrm{N}(A)} & =\operatorname{dim} \frac{\mathrm{N}_{A}^{\ell}(T)}{\mathrm{N}_{A}^{\ell}(T) \cap \mathrm{R}\left(T^{p}\right)+\mathrm{N}(A)} \\
& =\operatorname{dim} \frac{\mathrm{N}_{A}^{\ell}(T)}{\mathrm{N}_{A}^{\ell}(T) \cap\left[\mathrm{R}\left(T^{p}\right)+\mathrm{N}(A)\right]} \\
& =\operatorname{dim} \frac{\mathrm{N}_{A}^{\ell}\left(T^{p}\right)}{\mathrm{N}_{A}^{\ell}\left(T^{p}\right) \cap[\mathrm{R}(T)+\mathrm{N}(A)]}=\operatorname{dim} \frac{\mathrm{R}(T)+\mathrm{N}_{A}^{\ell}\left(T^{p}\right)}{\mathrm{R}(T)+\mathrm{N}(A)} .
\end{aligned}
$$

This leads to

$$
\operatorname{dim} \frac{\mathrm{N}_{A}^{\ell}(T)}{\mathrm{N}(A)} \leq \operatorname{dim} \frac{\mathrm{X}}{\mathrm{R}(T)+\mathrm{N}(A)}
$$

On the other hand, since $\alpha_{A}^{\ell}(T)=\operatorname{dim} \mathrm{N}_{A}^{\ell}(T) / \mathrm{N}(A)+\alpha(A)$, by Lemma 2.9 and (3.3), we obtain

$$
\begin{aligned}
\alpha_{A}^{\ell}(T)+\beta(A) & =\operatorname{dim} \frac{\mathrm{N}_{A}^{\ell}(T)}{\mathrm{N}(A)}+\alpha(A)+\beta(A) \\
& \leq \operatorname{dim} \frac{\mathrm{X}}{\mathrm{R}(T)+\mathrm{N}(A)}+\beta(A)+\alpha(A) \\
& =\beta_{A}^{\ell}(T)+\alpha(A) .
\end{aligned}
$$


Now, suppose that $\operatorname{des}_{A}^{\ell}(T)<+\infty$. Then, by Theorems 3.5 and 3.6, we get $\mathrm{X}=\mathrm{R}(T)+\mathrm{N}_{A}^{\ell}\left(T^{p}\right)$. Therefore, from (3.2), we see that

$$
\operatorname{dim} \frac{\mathrm{N}_{A}^{\ell}(T)}{\mathrm{N}(A)}=\operatorname{dim} \frac{\mathrm{X}}{\mathrm{R}(T)+\mathrm{N}(A)} .
$$

Arguing as in (3.4), with (3.5), we get the equality in (3.1).

Finally, suppose that there is equality in $(3.1), \max \{\alpha(A), \beta(A)\}<+\infty$ and $\min \left\{\alpha_{A}^{\ell}(T), \beta_{A}^{\ell}(T)\right\}<+\infty$. It follows from (3.2) and (3.4) that:

$$
\operatorname{dim} \frac{\mathrm{X}}{\mathrm{R}(T)+\mathrm{N}(A)}=\operatorname{dim} \frac{\mathrm{N}_{A}^{\ell}(T)}{\mathrm{N}(A)}=\operatorname{dim} \frac{\mathrm{R}(T)+\mathrm{N}_{A}^{\ell}\left(T^{p}\right)}{\mathrm{R}(T)+\mathrm{N}(A)}<+\infty,
$$

because $\operatorname{dim} \mathrm{N}_{A}^{\ell}(T) / \mathrm{N}(A) \leq \alpha_{A}^{\ell}(T)$ and $\operatorname{dim} \mathrm{X} /[\mathrm{R}(T)+\mathrm{N}(A)] \leq \beta_{A}^{\ell}(T)$ according to Lemma 2.9. This shows that $\mathrm{R}(T)+\mathrm{N}_{A}^{\ell}\left(T^{p}\right)=\mathrm{X}$, and hence, Theorem 3.5 implies that $\operatorname{des}_{A}^{\ell}(T)<+\infty$. The proof is complete.

Theorem 3.9 Let $A \in \mathscr{L}(\mathrm{X})$ and $T \in \mathscr{L}_{\mathrm{R}(A)}(\mathrm{X})$. If $p=\operatorname{asc}_{A}^{r}(T)<+\infty$, then

$$
\alpha_{A}^{r}(T)+\beta(A) \leq \beta_{A}^{r}(T)+\alpha(A),
$$

and there is equality in (3.6) if des $S_{A}^{r}(T)<+\infty$. Furthermore, if $\max \{\alpha(A), \beta(A)\}<+\infty, \min \left\{\alpha_{A}^{r}(T), \beta_{A}^{r}(T)\right\}$ $<+\infty$ and we have equality in (3.6), then $\operatorname{des}_{A}^{r}(T)<+\infty$.

Proof Since $p=\operatorname{asc}_{A}^{r}(T)<+\infty$, it follows from Theorem 3.4 that $\mathrm{N}(T) \cap \mathrm{R}_{A}^{r}\left(T^{p}\right)=\{0\}$. Therefore, by Remark 2.1 and Lemma 2.8, we infer that

$$
\operatorname{dim} \frac{\mathrm{N}_{A}^{r}(T)}{\mathrm{N}(A)}=\operatorname{dim} \mathrm{N}(T) \cap \mathrm{R}(A)=\operatorname{dim} \frac{\mathrm{N}(T) \cap \mathrm{R}(A)}{\mathrm{N}(T) \cap \mathrm{R}_{A}^{r}\left(T^{p}\right)}=\operatorname{dim} \frac{\mathrm{N}\left(T^{p}\right) \cap \mathrm{R}(A)}{\mathrm{N}\left(T^{p}\right) \cap \mathrm{R}_{A}^{r}(T)},
$$

and so, by [8, Lemma 2.2]

$$
\operatorname{dim} \frac{\mathrm{N}_{A}^{r}(T)}{\mathrm{N}(A)}=\operatorname{dim} \frac{\left(\mathrm{N}\left(T^{p}\right)+\mathrm{R}_{A}^{r}(T)\right) \cap \mathrm{R}(A)}{\mathrm{R}_{A}^{r}(T)} \leq \operatorname{dim} \frac{\mathrm{R}(A)}{\mathrm{R}_{A}^{r}(T)} .
$$

Since $\mathrm{N}(A) \subset \mathrm{N}_{A}^{r}(T)$ and $\mathrm{R}_{A}^{r}(T) \subset \mathrm{R}(A) \subset \mathrm{X}$, then (3.7) shows that

$$
\begin{aligned}
\alpha_{A}^{r}(T)+\beta(A) & =\operatorname{dim} \frac{\mathrm{N}_{A}^{r}(T)}{\mathrm{N}(A)}+\alpha(A)+\beta(A) \\
& \leq \operatorname{dim} \frac{\mathrm{R}(A)}{\mathrm{R}_{A}^{r}(T)}+\beta(A)+\alpha(A)=\beta_{A}^{r}(T)+\alpha(A) .
\end{aligned}
$$

If, additionally, $\operatorname{des}_{A}^{r}(T)<+\infty$, then Theorems 3.4 and 3.6 prove that $\mathrm{R}(A) \subset \mathrm{R}_{A}^{r}(T)+\mathrm{N}\left(T^{p}\right)$. Hence, (3.7) gives that $\operatorname{dim} \mathrm{N}_{A}^{r}(T) / \mathrm{N}(A)=\operatorname{dim} \mathrm{R}(A) / \mathrm{R}_{A}^{r}(T)$, and as in (3.8), we can easily check that $\alpha_{A}^{r}(T)+\beta(A)=$ $\beta_{A}^{r}(T)+\alpha(A)$.

Finally, suppose that there is equality in (3.6), $\max \{\alpha(A), \beta(A)\}<+\infty$ and $\min \left\{\alpha_{A}^{r}(T), \beta_{A}^{r}(T)\right\}<+\infty$. Therefore, the inequality in (3.8) gives

$$
\operatorname{dim} \frac{\mathrm{N}_{A}^{r}(T)}{\mathrm{N}(A)}=\operatorname{dim} \frac{\mathrm{R}(A)}{\mathrm{R}_{A}^{r}(T)} \leq \min \left\{\alpha_{A}^{r}(T), \beta_{A}^{r}(T)\right\}<+\infty .
$$

Hence, it follows by (3.7) that:

$$
\operatorname{dim} \frac{\left(\mathrm{N}\left(T^{p}\right)+\mathrm{R}_{A}^{r}(T)\right) \cap \mathrm{R}(A)}{\mathrm{R}_{A}^{r}(T)}=\operatorname{dim} \frac{\mathrm{R}(A)}{\mathrm{R}_{A}^{r}(T)}<+\infty .
$$

Therefore, $\left(\mathrm{N}\left(T^{p}\right)+\mathrm{R}_{A}^{r}(T)\right) \cap \mathrm{R}(A)=\mathrm{R}(A)$, i.e., $\mathrm{R}(A) \subset \mathrm{N}\left(T^{p}\right)+\mathrm{R}_{A}^{r}(T)$. Now, by Theorem 3.4, we get $\operatorname{des}_{A}^{r}(T)<+\infty$. This completes the proof.

Theorem 3.10 Let $A \in \mathscr{L}(\mathrm{X})$ and $T \in \mathscr{L}_{\mathrm{N}(A)}(\mathrm{X})$. Suppose that $\alpha_{A}^{\ell}(T)+\beta(A)=\beta_{A}^{\ell}(T)+\alpha(A)<+\infty$ and $p=\operatorname{asc}_{A}^{\ell}(T)<+\infty$. Then 
(1) $\operatorname{des}_{A}^{\ell}(T)=\operatorname{asc}_{A}^{\ell}(T)$;

(2) $\alpha_{A}^{\ell}\left(T^{i}\right)+\beta(A)=\beta_{A}^{\ell}\left(T^{i}\right)+\alpha(A)$, for $i=0,1, \cdots$.

Proof (1) follows from Theorems 3.6 and 3.8.

(2) Let $k$ be a non-negative integer. First of all, we observe that it follows from (1.1) that $\mathrm{R}_{A}^{\ell}\left(T^{k+1}\right) \subset$ $\mathrm{R}_{A}^{\ell}\left(T^{k}\right) \subset \mathrm{X}$, and hence

$$
\beta_{A}^{\ell}\left(T^{k+1}\right)=\beta_{A}^{\ell}\left(T^{k}\right)+\operatorname{dim} \frac{\mathrm{R}_{A}^{\ell}\left(T^{k}\right)}{\mathrm{R}_{A}^{\ell}\left(T^{k+1}\right)} .
$$

According to Lemma 2.10, $\beta_{A}^{\ell}\left(T^{k}\right)<+\infty$, because $\beta(A) \leq \beta_{A}^{\ell}(T)<+\infty$. Now, by Lemma 2.3, we can see the following:

$$
\beta_{A}^{\ell}\left(T^{k+1}\right)-\beta_{A}^{\ell}\left(T^{k}\right)=\operatorname{dim} \frac{\mathrm{X}}{\mathrm{R}(T)+\mathrm{N}_{A}^{\ell}\left(T^{k}\right)}, \quad \forall k \in \mathbb{N}
$$

On the other hand, since $\mathrm{R}(T)+\mathrm{N}(A) \subset \mathrm{R}(T)+\mathrm{N}_{A}^{\ell}\left(T^{k}\right) \subset \mathrm{X}$, by Lemma 2.9, we have

$$
\begin{aligned}
\beta_{A}^{\ell}(T)-\beta(A) & =\operatorname{dim} \frac{\mathrm{X}}{\mathrm{R}(T)+\mathrm{N}(A)} \\
& =\operatorname{dim} \frac{\mathrm{X}}{\mathrm{R}(T)+\mathrm{N}_{A}^{\ell}\left(T^{k}\right)}+\operatorname{dim} \frac{\mathrm{R}(T)+\mathrm{N}_{A}^{\ell}\left(T^{k}\right)}{\mathrm{R}(T)+\mathrm{N}(A)}<+\infty,
\end{aligned}
$$

and as $\operatorname{dim} \mathrm{N}_{A}^{\ell}(T) / \mathrm{N}(A)=\alpha_{A}^{\ell}(T)-\alpha(A)=\beta_{A}^{\ell}(T)-\beta(A)$, we get

$$
\beta_{A}^{\ell}\left(T^{k+1}\right)-\beta_{A}^{\ell}\left(T^{k}\right)=\operatorname{dim} \frac{\mathrm{X}}{\mathrm{R}(T)+\mathrm{N}_{A}^{\ell}\left(T^{k}\right)}=\operatorname{dim} \frac{\mathrm{N}_{A}^{\ell}(T)}{\mathrm{N}(A)}-\operatorname{dim} \frac{\mathrm{R}(T)+\mathrm{N}_{A}^{\ell}\left(T^{k}\right)}{\mathrm{R}(T)+\mathrm{N}(A)} .
$$

Now, [8, Lemma 2.2] and Lemma 2.6 show that

$$
\begin{aligned}
\operatorname{dim} \frac{\mathrm{R}(T)+\mathrm{N}_{A}^{\ell}\left(T^{k}\right)}{\mathrm{R}(T)+\mathrm{N}(A)} & =\operatorname{dim} \frac{\mathrm{N}_{A}^{\ell}\left(T^{k}\right)}{(\mathrm{R}(T)+\mathrm{N}(A)) \cap \mathrm{N}_{A}^{\ell}\left(T^{k}\right)} \\
& =\operatorname{dim} \frac{\mathrm{N}_{A}^{\ell}(T)}{\left(\mathrm{R}\left(T^{k}\right)+\mathrm{N}(A)\right) \cap \mathrm{N}_{A}^{\ell}(T)} .
\end{aligned}
$$

We note that $\mathrm{N}(A) \subset\left(\mathrm{R}\left(T^{k}\right)+\mathrm{N}(A)\right) \cap \mathrm{N}_{A}^{\ell}(T) \subset \mathrm{N}_{A}^{\ell}(T)$, and hence

$$
\operatorname{dim} \frac{\mathrm{N}_{A}^{\ell}(T)}{\mathrm{N}(A)}=\operatorname{dim} \frac{\mathrm{N}_{A}^{\ell}(T)}{\left(\mathrm{R}\left(T^{k}\right)+\mathrm{N}(A)\right) \cap \mathrm{N}_{A}^{\ell}(T)}+\operatorname{dim} \frac{\left(\mathrm{R}\left(T^{k}\right)+\mathrm{N}(A)\right) \cap \mathrm{N}_{A}^{\ell}(T)}{\mathrm{N}(A)}<+\infty
$$

Then, from (3.10) and [8, Lemma 2.2], it follows that:

$$
\begin{aligned}
\operatorname{dim} \frac{\mathrm{R}(T)+\mathrm{N}_{A}^{\ell}\left(T^{k}\right)}{\mathrm{R}(T)+\mathrm{N}(A)} & =\operatorname{dim} \frac{\mathrm{N}_{A}^{\ell}(T)}{\mathrm{N}(A)}-\operatorname{dim} \frac{\left(\mathrm{R}\left(T^{k}\right)+\mathrm{N}(A)\right) \cap \mathrm{N}_{A}^{\ell}(T)}{\mathrm{N}(A)} \\
& \left.=\operatorname{dim} \frac{\mathrm{N}_{A}^{\ell}(T)}{\mathrm{N}(A)}\right)-\operatorname{dim} \frac{\mathrm{R}\left(T^{k}\right) \cap \mathrm{N}_{A}^{\ell}(T)+\mathrm{N}(A)}{\mathrm{N}(A)} \\
& =\operatorname{dim} \frac{\mathrm{N}_{A}^{\ell}(T)}{\mathrm{N}(A)}-\operatorname{dim} \frac{\mathrm{R}\left(T^{k}\right) \cap \mathrm{N}_{A}^{\ell}(T)}{\mathrm{R}\left(T^{k}\right) \cap \mathrm{N}(A)}
\end{aligned}
$$

Hence, by (3.9) and Lemma 2.2, we infer that

$$
\beta_{A}^{\ell}\left(T^{k+1}\right)-\beta_{A}^{\ell}\left(T^{k}\right)=\operatorname{dim} \frac{\mathrm{R}\left(T^{k}\right) \cap \mathrm{N}_{A}^{\ell}(T)}{\mathrm{R}\left(T^{k}\right) \cap \mathrm{N}(A)}=\operatorname{dim} \frac{\mathrm{N}_{A}^{\ell}\left(T^{k+1}\right)}{\mathrm{N}_{A}^{\ell}\left(T^{k}\right)} .
$$


Since $\alpha_{A}^{\ell}(T)<+\infty$, we have $\alpha_{A}^{\ell}\left(T^{k}\right) \leq \alpha_{A}^{\ell}\left(T^{k+1}\right)<+\infty$ (see Lemma 2.10), and so

$$
\beta_{A}^{\ell}\left(T^{k+1}\right)-\beta_{A}^{\ell}\left(T^{k}\right)=\alpha_{A}^{\ell}\left(T^{k+1}\right)-\alpha_{A}^{\ell}\left(T^{k}\right), \quad \forall k \in \mathbb{N} .
$$

Hence, $\alpha_{A}^{\ell}\left(T^{0}\right)=\alpha(A)$ and $\beta_{A}^{\ell}\left(T^{0}\right)=\beta(A)$ imply

$$
\begin{aligned}
\beta_{A}^{\ell}\left(T^{i}\right)-\beta(A) & =\sum_{k=0}^{i-1}\left(\beta_{A}^{\ell}\left(T^{k+1}\right)-\beta_{A}^{\ell}\left(T^{k}\right)\right) \\
& =\sum_{k=0}^{i-1}\left(\alpha_{A}^{\ell}\left(T^{k+1}\right)-\alpha_{A}^{\ell}\left(T^{k}\right)\right)=\alpha_{A}^{\ell}\left(T^{i}\right)-\alpha(A), \quad \forall i \geq 1 .
\end{aligned}
$$

The proof is complete.

Theorem 3.11 Let $A \in \mathscr{L}(\mathrm{X})$ and $T \in \mathscr{L}_{\mathrm{R}(A)}(\mathrm{X})$. Suppose that $\alpha_{A}^{r}(T)+\beta(A)=\beta_{A}^{r}(T)+\alpha(A)<+\infty$ and $p=\operatorname{asc}_{A}^{r}(T)<+\infty$. Then

(1) $\operatorname{des}_{A}^{r}(T)=\operatorname{asc}_{A}^{r}(T)$,

(2) $\alpha_{A}^{r}\left(T^{i}\right)+\beta(A)=\beta_{A}^{r}\left(T^{i}\right)+\alpha(A)$, for $i=0,1, \cdots$.

Proof (1) follows from Theorems 3.6 and 3.9.

(2) Let $k$ be a non-negative integer. From $\mathrm{R}_{A}^{r}\left(T^{k}\right) \subset \mathrm{R}_{A}^{r}\left(T^{k+1}\right) \subset \mathrm{X}$, it follows that

$$
\beta_{A}^{r}\left(T^{k+1}\right)=\beta_{A}^{r}\left(T^{k}\right)+\operatorname{dim} \frac{\mathrm{R}_{A}^{r}\left(T^{k}\right)}{\mathrm{R}_{A}^{r}\left(T^{k+1}\right)} .
$$

Since $\beta_{A}^{r}(T)<+\infty$, by Lemma 2.10, we have $\beta_{A}^{r}\left(T^{k}\right) \leq \beta_{A}^{r}\left(T^{k+1}\right)<+\infty$, and so

$$
\beta_{A}^{r}\left(T^{k+1}\right)-\beta_{A}^{r}\left(T^{k}\right)=\operatorname{dim} \frac{\mathrm{R}_{A}^{r}\left(T^{k}\right)}{\mathrm{R}_{A}^{r}\left(T^{k+1}\right)} .
$$

Then, we deduce from Lemma 2.3 that

$$
\beta_{A}^{r}\left(T^{k+1}\right)-\beta_{A}^{r}\left(T^{k}\right)=\operatorname{dim} \frac{\mathrm{R}(A)}{\left(\mathrm{R}_{A}^{r}(T)+\mathrm{N}\left(T^{k}\right)\right) \cap \mathrm{R}(A)} .
$$

Since $\mathrm{R}_{A}^{r}(T) \subset\left(\mathrm{R}_{A}^{r}(T)+\mathrm{N}\left(T^{k}\right)\right) \cap \mathrm{R}(A) \subset \mathrm{R}(A)$ and $\mathrm{R}_{A}^{r}(T) \subset \mathrm{R}(A) \subset \mathrm{X}$, it follows that:

$$
\begin{aligned}
+\infty>\beta_{A}^{r}(T)-\beta(A) & =\operatorname{dim} \frac{\mathrm{R}(A)}{\mathrm{R}_{A}^{r}(T)} \\
& =\operatorname{dim} \frac{\mathrm{R}(A)}{\left(\mathrm{R}_{A}^{r}(T)+\mathrm{N}\left(T^{k}\right)\right) \cap \mathrm{R}(A)}+\operatorname{dim} \frac{\left(\mathrm{R}_{A}^{r}(T)+\mathrm{N}\left(T^{k}\right)\right) \cap \mathrm{R}(A)}{\mathrm{R}_{A}^{r}(T)} .
\end{aligned}
$$

Combining this with formula (3.11), we obtain

$$
\beta_{A}^{r}\left(T^{k+1}\right)-\beta_{A}^{r}\left(T^{k}\right)=\beta_{A}^{r}(T)-\beta(A)-\operatorname{dim} \frac{\left(\mathrm{R}_{A}^{r}(T)+\mathrm{N}\left(T^{k}\right)\right) \cap \mathrm{R}(A)}{\mathrm{R}_{A}^{r}(T)} .
$$

Observe that [8, Lemma 2.2] and Lemma 2.8 imply that

$$
\begin{aligned}
\operatorname{dim} \frac{\left(\mathrm{R}_{A}^{r}(T)+\mathrm{N}\left(T^{k}\right)\right) \cap \mathrm{R}(A)}{\mathrm{R}_{A}^{r}(T)} & =\operatorname{dim} \frac{\mathrm{R}_{A}^{r}(T)+\mathrm{N}\left(T^{k}\right) \cap \mathrm{R}(A)}{\mathrm{R}_{A}^{r}(T)} \\
& =\operatorname{dim} \frac{\mathrm{N}\left(T^{k}\right) \cap \mathrm{R}(A)}{\mathrm{N}\left(T^{k}\right) \cap \mathrm{R}_{A}^{r}(T)}=\operatorname{dim} \frac{\mathrm{N}(T) \cap \mathrm{R}(A)}{\mathrm{N}(T) \cap \mathrm{R}_{A}^{r}\left(T^{k}\right)}
\end{aligned}
$$


Since $\alpha_{A}^{r}(T)=\operatorname{dim} \mathrm{N}_{A}^{r}(T) / \mathrm{N}(A)+\alpha(A)$ and $\mathrm{N}(T) \cap \mathrm{R}_{A}^{r}\left(T^{k}\right) \subset \mathrm{N}(T) \cap \mathrm{R}(A)$, by Remark 2.1 and Lemma 2.2, we have

$$
\begin{aligned}
+\infty>\alpha_{A}^{r}(T)-\alpha(A) & =\operatorname{dim} \frac{\mathrm{N}_{A}^{r}(T)}{\mathrm{N}(A)}=\operatorname{dim} \mathrm{N}(T) \cap \mathrm{R}(A) \\
& =\operatorname{dim} \frac{\mathrm{N}(T) \cap \mathrm{R}(A)}{\mathrm{N}(T) \cap \mathrm{R}_{A}^{r}\left(T^{k}\right)}+\operatorname{dim} \mathrm{N}(T) \cap \mathrm{R}_{A}^{r}\left(T^{k}\right) \\
& =\operatorname{dim} \frac{\mathrm{N}(T) \cap \mathrm{R}(A)}{\mathrm{N}(T) \cap \mathrm{R}_{A}^{r}\left(T^{k}\right)}+\operatorname{dim} \frac{\mathrm{N}_{A}^{r}\left(T^{k+1}\right)}{\mathrm{N}_{A}^{r}\left(T^{k}\right)} .
\end{aligned}
$$

Combining these facts with formula (3.12) and using the hypothesis $\alpha_{A}^{r}(T)+\beta(A)=\beta_{A}^{r}(T)+\alpha(A)<+\infty$, we obtain

$$
\beta_{A}^{r}\left(T^{k+1}\right)-\beta_{A}^{r}\left(T^{k}\right)=\operatorname{dim} \frac{\mathrm{N}_{A}^{r}\left(T^{k+1}\right)}{\mathrm{N}_{A}^{r}\left(T^{k}\right)} .
$$

Since $\mathrm{N}_{A}^{r}\left(T^{k}\right) \subset \mathrm{N}_{A}^{r}\left(T^{k+1}\right)$ and since $\alpha_{A}^{r}\left(T^{k+1}\right)<+\infty$ according to Lemma 2.10, it follows that:

$$
\beta_{A}^{r}\left(T^{k+1}\right)-\beta_{A}^{r}\left(T^{k}\right)=\alpha_{A}^{r}\left(T^{k+1}\right)-\alpha_{A}^{r}\left(T^{k}\right), \quad \forall k \in \mathbb{N} .
$$

Hence, $\beta_{A}^{r}\left(T^{0}\right)=\beta(A)$ and $\alpha_{A}^{r}\left(T^{0}\right)=\alpha(A)$ imply

$$
\begin{aligned}
\beta_{A}^{r}\left(T^{i}\right)-\beta(A) & =\sum_{k=0}^{i-1}\left(\beta_{A}^{r}\left(T^{k+1}\right)-\beta_{A}^{r}\left(T^{k}\right)\right) \\
& =\sum_{k=0}^{i-1}\left(\alpha_{A}^{r}\left(T^{k+1}\right)-\alpha_{A}^{r}\left(T^{k}\right)\right)=\alpha_{A}^{r}\left(T^{i}\right)-\alpha(A), \quad \forall i \geq 1 .
\end{aligned}
$$

The proof is complete.

Theorem 3.12 Let $A \in \mathscr{L}(\mathrm{X})$ and $T \in \mathscr{L}_{\mathrm{N}(A)}(\mathrm{X})$. If $q=\operatorname{des}_{A}^{\ell}(T)<+\infty$, then

$$
\beta_{A}^{\ell}(T)+\alpha(A) \leq \alpha_{A}^{\ell}(T)+\beta(A),
$$

and there is equality in (3.13) if $\operatorname{asc}_{A}^{\ell}(T)<+\infty$. Furthermore, if $\max \{\alpha(A), \beta(A)\}<+\infty$, $\min \left\{\alpha_{A}^{\ell}(T), \beta_{A}^{\ell}(T)\right\}<+\infty$ and we have equality in (3.13), then $\operatorname{asc}_{A}^{\ell}(T)<+\infty$.

Proof Since $q=\operatorname{des}_{A}^{\ell}(T)<+\infty$, by Lemma 2.3, we have $\mathrm{X}=\mathrm{R}(T)+\mathrm{N}_{A}^{\ell}\left(T^{q}\right)$. Then, Remark 2.1 and [8, Lemma 2.2] show that

$$
\begin{aligned}
\operatorname{dim} \frac{\mathrm{R}(A)}{\mathrm{R}_{A}^{\ell}(T)} & =\operatorname{dim} \frac{\mathrm{X}}{\mathrm{R}(T)+\mathrm{N}(A)} \\
& =\operatorname{dim} \frac{\mathrm{R}(T)+\mathrm{N}_{A}^{\ell}\left(T^{q}\right)}{\mathrm{R}(T)+\mathrm{N}(A)}=\operatorname{dim} \frac{\mathrm{N}_{A}^{\ell}\left(T^{q}\right)}{(\mathrm{R}(T)+\mathrm{N}(A)) \cap \mathrm{N}_{A}^{\ell}\left(T^{q}\right)} .
\end{aligned}
$$

But then, Lemma 2.6 implies that

$$
\operatorname{dim} \frac{\mathrm{R}(A)}{\mathrm{R}_{A}^{\ell}(T)}=\operatorname{dim} \frac{\mathrm{N}_{A}^{\ell}(T)}{\left(\mathrm{R}\left(T^{q}\right)+\mathrm{N}(A)\right) \cap \mathrm{N}_{A}^{\ell}(T)},
$$

and as $\mathrm{N}(A) \subset\left(\mathrm{R}\left(T^{q}\right)+\mathrm{N}(A)\right) \cap \mathrm{N}_{A}^{\ell}(T)$, we get

$$
\operatorname{dim} \frac{\mathrm{R}(A)}{\mathrm{R}_{A}^{\ell}(T)} \leq \operatorname{dim} \frac{\mathrm{N}_{A}^{\ell}(T)}{\mathrm{N}(A)} .
$$


Furthermore, since $\mathrm{R}_{A}^{\ell}(T) \subset \mathrm{R}(A) \subset \mathrm{X}$ and $\mathrm{N}(A) \subset \mathrm{N}_{A}^{\ell}(T)$, it follows that:

$$
\begin{aligned}
\beta_{A}^{\ell}(T)+\alpha(A) & =\operatorname{dim} \frac{\mathrm{R}(A)}{\mathrm{R}_{A}^{\ell}(T)}+\beta(A)+\alpha(A) \\
& \leq \operatorname{dim} \frac{\mathrm{N}_{A}^{\ell}(T)}{\mathrm{N}(A)}+\alpha(A)+\beta(A)=\alpha_{A}^{\ell}(T)+\beta(A) .
\end{aligned}
$$

If, in addition, $\operatorname{asc}_{A}^{\ell}(T)<+\infty$, by Theorem 3.6, $\operatorname{asc}_{A}^{\ell}(T)=q$ and by Theorem 3.5, we deduce that

$$
\left(\mathrm{R}\left(T^{q}\right)+\mathrm{N}(A)\right) \cap \mathrm{N}_{A}^{\ell}(T)=\mathrm{R}\left(T^{q}\right) \cap \mathrm{N}_{A}^{\ell}(T)+\mathrm{N}(A)=\mathrm{N}(A) .
$$

Therefore, (3.14) implies that $\operatorname{dim} \mathrm{R}(A) / \mathrm{R}_{A}^{\ell}(T)=\operatorname{dim} \mathrm{N}_{A}^{\ell}(T) / \mathrm{N}(A)$, and hence, we can prove that $\beta_{A}^{\ell}(T)+$ $\alpha(A)=\alpha_{A}^{\ell}(T)+\beta(A)$ similarly as in (3.15).

Conversely, suppose that $\alpha_{A}^{\ell}(T)+\beta(A)=\beta_{A}^{\ell}(T)+\alpha(A), \max \{\alpha(A), \beta(A)\}<+\infty$ and $\min \left\{\alpha_{A}^{\ell}(T)\right.$, $\left.\beta_{A}^{\ell}(T)\right\}<+\infty$. Then, by (3.15), we obtain that

$$
\operatorname{dim} \frac{\mathrm{N}_{A}^{\ell}(T)}{\mathrm{N}(A)}=\operatorname{dim} \frac{\mathrm{R}(A)}{\mathrm{R}_{A}^{\ell}(T)} \leq \min \left\{\alpha_{A}^{\ell}(T), \beta_{A}^{\ell}(T)\right\}<+\infty,
$$

and so, (3.14) gives

$$
\operatorname{dim} \frac{\mathrm{N}_{A}^{\ell}(T)}{\mathrm{N}(A)}=\operatorname{dim} \frac{\mathrm{N}_{A}^{\ell}(T)}{\left(\mathrm{R}\left(T^{q}\right)+\mathrm{N}(A)\right) \cap \mathrm{N}_{A}^{\ell}(T)}<+\infty .
$$

Since $\mathrm{N}(A) \subset\left(\mathrm{R}\left(T^{q}\right)+\mathrm{N}(A)\right) \cap \mathrm{N}_{A}^{\ell}(T) \subset \mathrm{N}_{A}^{\ell}(T)$, it follows that:

$$
\operatorname{dim} \frac{\left(\mathrm{R}\left(T^{q}\right)+\mathrm{N}(A)\right) \cap \mathrm{N}_{A}^{\ell}(T)}{\mathrm{N}(A)}=\operatorname{dim} \frac{\mathrm{N}_{A}^{\ell}(T)}{\mathrm{N}(A)}-\operatorname{dim} \frac{\mathrm{N}_{A}^{\ell}(T)}{\left(\mathrm{R}\left(T^{q}\right)+\mathrm{N}(A)\right) \cap \mathrm{N}_{A}^{\ell}(T)}=0,
$$

and as a consequence of Lemma 2.2 and [8, Lemma 2.2], we have

$$
\begin{aligned}
\operatorname{dim} \frac{\mathrm{N}_{A}^{\ell}\left(T^{q+1}\right)}{\mathrm{N}_{A}^{\ell}\left(T^{q}\right)} & =\operatorname{dim} \frac{\mathrm{R}\left(T^{q}\right) \cap \mathrm{N}_{A}^{\ell}(T)}{\mathrm{R}\left(T^{q}\right) \cap \mathrm{N}(A)} \\
& =\operatorname{dim} \frac{\mathrm{R}\left(T^{q}\right) \cap \mathrm{N}_{A}^{\ell}(T)+\mathrm{N}(A)}{\mathrm{N}(A)}=\operatorname{dim} \frac{\left(\mathrm{R}\left(T^{q}\right)+\mathrm{N}(A)\right) \cap \mathrm{N}_{A}^{\ell}(T)}{\mathrm{N}(A)}=0 .
\end{aligned}
$$

This implies that $\operatorname{asc}_{A}^{\ell}(T)<+\infty$ and the proof is complete.

Theorem 3.13 Let $A \in \mathscr{L}(\mathrm{X})$ and $T \in \mathscr{L}_{\mathrm{R}(A)}(\mathrm{X})$. If $q=\operatorname{des}_{A}^{r}(T)<+\infty$, then

$$
\beta_{A}^{r}(T)+\alpha(A) \leq \alpha_{A}^{r}(T)+\beta(A),
$$

and there is equality in (3.16) if $\operatorname{asc}_{A}^{r}(T)<+\infty$. Furthermore, if $\max \{\alpha(A), \beta(A)\}<+\infty$, $\min \left\{\alpha_{A}^{r}(T), \beta_{A}^{r}(T)\right\}<+\infty$ and we have equality in (3.16), then $\operatorname{asc}_{A}^{r}(T)<+\infty$.

Proof From Theorem 3.4, we have $\mathrm{R}(A) \subset \mathrm{R}_{A}^{r}(T)+\mathrm{N}\left(T^{q}\right)$, and so by [8, Lemma 2.2] and Lemma 2.8, it follows that:

$$
\begin{aligned}
\operatorname{dim} \frac{\mathrm{R}(A)}{\mathrm{R}_{A}^{r}(T)} & =\operatorname{dim} \frac{\left(\mathrm{R}_{A}^{r}(T)+\mathrm{N}\left(T^{q}\right)\right) \cap \mathrm{R}(A)}{\mathrm{R}_{A}^{r}(T)}=\operatorname{dim} \frac{\mathrm{R}_{A}^{r}(T)+\mathrm{N}\left(T^{q}\right) \cap \mathrm{R}(A)}{\mathrm{R}_{A}^{r}(T)} \\
& =\operatorname{dim} \frac{\mathrm{R}(A) \cap \mathrm{N}\left(T^{q}\right)}{\mathrm{R}_{A}^{r}(T) \cap \mathrm{N}\left(T^{q}\right)}=\operatorname{dim} \frac{\mathrm{R}(A) \cap \mathrm{N}(T)}{\mathrm{R}_{A}^{r}\left(T^{q}\right) \cap \mathrm{N}(T)}
\end{aligned}
$$


Since $\mathrm{R}_{A}^{r}(T) \subset \mathrm{R}(A) \subset \mathrm{X}$ and $\mathrm{N}(A) \subset \mathrm{N}_{A}^{r}(T)$, then $\beta_{A}^{r}(T)=\operatorname{dim} \mathrm{R}(A) / \mathrm{R}_{A}^{r}(T)+\beta(A)$ and $\alpha_{A}^{r}(T)=$ $\operatorname{dim} \mathrm{N}_{A}^{r}(T) / \mathrm{N}(A)+\alpha(A)$. Hence, by (3.17) and Remark 2.1, we infer that

$$
\begin{aligned}
\beta_{A}^{r}(T)+\alpha(A) & =\operatorname{dim} \frac{\mathrm{R}(A)}{\mathrm{R}_{A}^{r}(T)}+\beta(A)+\alpha(A) \\
& =\operatorname{dim} \frac{\mathrm{R}(A) \cap \mathrm{N}(T)}{\mathrm{R}_{A}^{r}\left(T^{q}\right) \cap \mathrm{N}(T)}+\beta(A)+\alpha(A) \\
& \leq \operatorname{dim} \mathrm{R}(A) \cap \mathrm{N}(T)+\beta(A)+\alpha(A) \\
& \leq \operatorname{dim} \frac{\mathrm{N}_{A}^{r}(T)}{\mathrm{N}(A)}+\alpha(A)+\beta(A)=\alpha_{A}^{r}(T)+\beta(A)
\end{aligned}
$$

Now, if $\operatorname{asc}_{A}^{r}(T)<+\infty$, then from Theorems 3.4 and 3.6, $\mathrm{N}(T) \cap \mathrm{R}_{A}^{r}\left(T^{q}\right)=\{0\}$. Therefore, (3.17) shows that $\operatorname{dim} \mathrm{R}(A) / \mathrm{R}_{A}^{r}(T)=\operatorname{dim} \mathrm{R}(A) \cap \mathrm{N}(T)$, and as in (3.18), we can prove that $\beta_{A}^{r}(T)+\alpha(A)=\alpha_{A}^{r}(T)+\beta(A)$.

Finally, assume that $\max \{\alpha(A), \beta(A)\}<+\infty, \min \left\{\alpha_{A}^{r}(T), \beta_{A}^{r}(T)\right\}<+\infty$ and we have equality in (3.16). Next, it is shown that $\operatorname{asc}_{A}^{r}(T)<+\infty$. From (3.18), it follows that:

$$
\operatorname{dim} \frac{\mathrm{R}(A)}{\mathrm{R}_{A}^{r}(T)}=\operatorname{dim} \mathrm{R}(A) \cap \mathrm{N}(T)=\operatorname{dim} \frac{\mathrm{N}_{A}^{r}(T)}{\mathrm{N}(A)} \leq \min \left\{\alpha_{A}^{r}(T), \beta_{A}^{r}(T)\right\}<+\infty,
$$

and by (3.17), we get

$$
\operatorname{dim} \mathrm{R}(A) \cap \mathrm{N}(T)=\operatorname{dim} \frac{\mathrm{R}(A)}{\mathrm{R}_{A}^{r}(T)}=\operatorname{dim} \frac{\mathrm{R}(A) \cap \mathrm{N}(T)}{\mathrm{R}_{A}^{r}\left(T^{q}\right) \cap \mathrm{N}(T)}<+\infty .
$$

Consequently, $\mathrm{R}_{A}^{r}\left(T^{q}\right) \cap \mathrm{N}(T)=\{0\}$ and Theorem 3.4 implies that $\operatorname{asc}_{A}^{r}(T)<+\infty$. This completes the proof.

\section{Compact perturbations}

In this section, some known results related to the stability of the ascent and descent of an operator [9, Sections 2 and 3] are extended to $A$-left (resp., $A$-right) ascent and $A$-left (resp., $A$-right) descent.

We start our study with the following algebraic results for later use.

Lemma 4.1 Let $A \in \mathscr{L}(\mathrm{X})$ and $C, T \in \mathscr{L}_{\mathrm{N}(A)}(\mathrm{X})$. Suppose that $C$ commutes with $T$. Then, for $n, k \in \mathbb{N} \backslash\{0\}$

$$
\begin{aligned}
\operatorname{dim} \frac{\mathrm{N}_{A}^{\ell}\left(T^{n}\right)}{\mathrm{N}_{A}^{\ell}\left[(T+C)^{n+k-1}\right] \cap \mathrm{N}_{A}^{\ell}\left(T^{n}\right)} & \leq \operatorname{dim} \mathrm{R}_{A}^{\ell}\left(C^{k}\right) ; \\
\operatorname{dim} \frac{\mathrm{R}_{A}^{\ell}\left(T^{n+k-1}\right)}{\mathrm{R}_{A}^{\ell}\left[(T+C)^{n}\right] \cap \mathrm{R}_{A}^{\ell}\left(T^{n+k-1}\right)} & \leq \operatorname{dim} \mathrm{R}_{A}^{\ell}\left(C^{k}\right) .
\end{aligned}
$$

Proof Let $n, k \in \mathbb{N} \backslash\{0\}$ and $x \in \mathbf{N}_{A}^{\ell}\left(T^{n}\right)$, and then, there exist $\alpha_{1}, \cdots, \alpha_{n+k-1} \in \mathbb{N}$, such that

$$
\begin{aligned}
A(T+C)^{n+k-1} x & =A\left(\sum_{i=0}^{n+k-1} \alpha_{i} T^{i} C^{n+k-1-i} x\right) \\
& =A\left(\sum_{i=0}^{n-1} \alpha_{i} T^{i} C^{n+k-1-i} x\right)+A\left(\sum_{i=n}^{n+k-1} \alpha_{i} T^{i} C^{n+k-1-i} x\right) \\
& =A C^{k}\left(\sum_{i=0}^{n-1} \alpha_{i} T^{i} C^{n-1-i} x\right)+\sum_{i=n}^{n+k-1} \alpha_{i} A T^{i} C^{n+k-1-i} x
\end{aligned}
$$

On the other hand, by (1.3), we have $x \in \mathrm{N}_{A}^{\ell}\left(T^{n}\right) \subset \mathrm{N}_{A}^{\ell}\left(T^{i}\right)$, for all $i \geq n$, this implies that $T^{i} x \in \mathrm{N}(A)$, and as $C(\mathrm{~N}(A)) \subset \mathrm{N}(A)$, we infer that $C^{n+k-1-i} T^{i} x \in \mathrm{N}(A)$. Thus

$$
A T^{i} C^{n+k-1-i} x=A C^{n+k-1-i} T^{i} x=0,
$$


and hence

$$
A(T+C)^{n+k-1} x=A C^{k}\left(\sum_{i=0}^{n-1} \alpha_{i} T^{i} C^{n-1-i} x\right) \in \mathrm{R}_{A}^{\ell}\left(C^{k}\right) .
$$

It follows from this that $J(x)=A(T+C)^{n+k-1}(x)$ defines a linear operator from $\mathrm{N}_{A}^{\ell}\left(T^{n}\right)$ into $\mathrm{R}_{A}^{\ell}\left(C^{k}\right)$, and as $\mathrm{N}(J)=\mathrm{N}_{A}^{\ell}\left[(T+C)^{n+k-1}\right] \cap \mathrm{N}_{A}^{\ell}\left(T^{n}\right)$, we obtain

$$
\operatorname{dim} \frac{\mathrm{N}_{A}^{\ell}\left(T^{n}\right)}{\mathrm{N}_{A}^{\ell}\left[(T+C)^{n+k-1}\right] \cap \mathrm{N}_{A}^{\ell}\left(T^{n}\right)} \leq \operatorname{dim} \mathrm{R}_{A}^{\ell}\left(C^{k}\right) .
$$

Now, let us show the second inequality in Lemma 4.1. Let $x_{1}, \cdots, x_{m} \in \mathrm{X}$, such that $A T^{n+k-1} x_{1}, \cdots$, $A T^{n+k-1} x_{m}$ are linearly independent in $\mathrm{R}_{A}^{\ell}\left(T^{n+k-1}\right)$ modulo $\mathrm{R}_{A}^{\ell}\left[(T+C)^{n}\right] \cap \mathrm{R}_{A}^{\ell}\left(T^{n+k-1}\right)$. Since $-C$ commutes with $T+C$, it follows that, for $i=1, \cdots, m$

$$
A T^{n+k-1} x_{i}=A(T+C)^{n} u_{i}+A C^{k} v_{i}
$$

for suitable $u_{i}$ and $v_{i}$. If $m>\operatorname{dim} \mathrm{R}_{A}^{\ell}\left(C^{k}\right)$, then there exist constants $a_{1}, \cdots, a_{m}$ not all zero, such that $\sum_{i=1}^{m} a_{i} A C^{k} v_{i}=0$, and hence

$$
\sum_{i=1}^{m} a_{i} A T^{n+k-1} x_{i}=\sum_{i=1}^{m} a_{i} A(T+C)^{n} u_{i} .
$$

Since the $a_{i}$ are not all zero, $\left\{A T^{n+k-1} x_{i}\right\}_{i=1}^{m}$ is not linearly independent modulo $\mathrm{R}_{A}^{\ell}\left[(T+C)^{n}\right] \cap \mathrm{R}_{A}^{\ell}\left(T^{n+k-1}\right)$, a contradiction. Thus, $m \leq \operatorname{dim} \mathbf{R}_{A}^{\ell}\left(C^{k}\right)$, which completes the proof.

Lemma 4.2 Let $A \in \mathscr{L}(\mathrm{X})$ and $C, T \in \mathscr{L}_{\mathrm{R}(A)}(\mathrm{X})$. Suppose that $C$ commutes with $T$. Then, for $n, k \in \mathbb{N} \backslash\{0\}$

$$
\begin{aligned}
\operatorname{dim} \frac{\mathrm{N}_{A}^{r}\left(T^{n}\right)}{\mathrm{N}_{A}^{r}\left[(T+C)^{n+k-1}\right] \cap \mathrm{N}_{A}^{r}\left(T^{n}\right)} & \leq \operatorname{dim} \mathrm{R}_{A}^{r}\left(C^{k}\right) ; \\
\operatorname{dim} \frac{\mathrm{R}_{A}^{r}\left(T^{n+k-1}\right)}{\mathrm{R}_{A}^{r}\left[(T+C)^{n}\right] \cap \mathrm{R}_{A}^{r}\left(T^{n+k-1}\right)} & \leq \operatorname{dim} \mathrm{R}_{A}^{r}\left(C^{k}\right) .
\end{aligned}
$$

Proof Let $n, k \in \mathbb{N} \backslash\{0\}$ and $x \in \mathbb{N}_{A}^{r}\left(T^{n}\right)$. Then, there exist constants $\alpha_{1}, \cdots, \alpha_{n+k-1}$, such that

$$
\begin{aligned}
(T+C)^{n+k-1} A x & =\left(\sum_{i=0}^{n+k-1} \alpha_{i} T^{i} C^{n+k-1-i}\right) A x \\
& =\left(\sum_{i=0}^{n-1} \alpha_{i} T^{i} C^{n+k-1-i}\right) A x+\left(\sum_{i=n}^{n+k-1} \alpha_{i} T^{i} C^{n+k-1-i}\right) A x \\
& =C^{k}\left(\sum_{i=0}^{n-1} \alpha_{i} T^{i} C^{n-1-i}\right) A x+\left(\sum_{i=n}^{n+k-1} \alpha_{i} C^{n+k-1-i} T^{i} A x\right) .
\end{aligned}
$$

However, by (1.1), we have $\mathrm{N}_{A}^{r}\left(T^{n}\right) \subset \mathrm{N}_{A}^{r}\left(T^{i}\right)$, for all $i \geq n$, and this implies that

$$
(T+C)^{n+k-1} A x=C^{k}\left(\sum_{i=0}^{n-1} \alpha_{i} C^{n-1-i} T^{i} A x\right) \in \mathrm{R}_{A}^{r}\left(C^{k}\right),
$$

because $C^{m} T^{j}(\mathrm{R}(A)) \subset \mathrm{R}(A)$, for all $j, m \in \mathbb{N}$. It follows from this that $J(x)=(T+C)^{n+k-1} A(x)$ defines a linear operator from $\mathrm{N}_{A}^{r}\left(T^{n}\right)$ into $\mathrm{R}_{A}^{r}\left(C^{k}\right)$, and as $\mathrm{N}(J)=\mathrm{N}_{A}^{r}\left[(T+C)^{n+k-1}\right] \cap \mathrm{N}_{A}^{r}\left(T^{n}\right)$, we obtain the first inequality in Lemma 4.2. 
To prove the second inequality in Lemma 4.2, let $x_{1}, \cdots, x_{m} \in \mathrm{X}$, such that $T^{n+k-1} A x_{1}, \cdots, T^{n+k-1} A x_{m}$ are linearly independent in $\mathrm{R}_{A}^{r}\left(T^{n+k-1}\right)$ modulo $\mathrm{R}_{A}^{r}\left[(T+C)^{n}\right] \cap \mathrm{R}_{A}^{r}\left(T^{n+k-1}\right)$. Since $-C$ commutes with $T+C$ and $C, T+C \in \mathscr{L}_{\mathrm{R}(A)}(\mathrm{X})$, it follows that, for $i=1, \cdots, m$

$$
\begin{aligned}
T^{n+k-1} A x_{i} & =((T+C)-C)^{n+k-1} A x_{i} \\
& =\sum_{j=0}^{n+k-1} \alpha_{j}(T+C)^{j} C^{n+k-1-j} A x_{i}, \quad \text { for some constants } \alpha_{j} \\
& =\sum_{j=0}^{n-1} \alpha_{j}(T+C)^{j} C^{n+k-1-j} A x_{i}+\sum_{j=n}^{n+k-1} \alpha_{j}(T+C)^{j} C^{n+k-1-j} A x_{i} \\
& =C^{k} \sum_{j=0}^{n-1} \underbrace{\alpha_{j}(T+C)^{j} C^{n-1-j} A x_{i}}_{\in \mathrm{R}(A)}+(T+C)^{n} \sum_{j=n}^{n+k-1} \underbrace{\alpha_{j}(T+C)^{j-n} C^{n+k-1-j} A x_{i}}_{\in \mathrm{R}(A)} .
\end{aligned}
$$

Hence

$$
T^{n+k-1} A x_{i}=(T+C)^{n} A u_{i}+C^{k} A v_{i}
$$

for suitable $u_{i}$ and $v_{i}$. If $m>\operatorname{dim} \mathrm{R}_{A}^{r}\left(C^{k}\right)$, then there exist constants $a_{1}, \cdots, a_{m}$ not all zero, such that $\sum_{i=1}^{m} a_{i} C^{k} A v_{i}=0$, and hence

$$
\sum_{i=1}^{m} a_{i} T^{n+k-1} A x_{i}=\sum_{i=1}^{m} a_{i}(T+C)^{n} A u_{i} .
$$

Since the $a_{i}$ are not all zero, $\left\{T^{n+k-1} A x_{i}\right\}_{i=1}^{m}$ is not linearly independent modulo $\mathrm{R}_{A}^{r}\left[(T+C)^{n}\right] \cap \mathrm{R}_{A}^{r}\left(T^{n+k-1}\right)$, a contradiction. Thus, $m \leq \operatorname{dim} \mathrm{R}_{A}^{r}\left(C^{k}\right)$, which proves that

$$
\operatorname{dim} \frac{\mathrm{R}_{A}^{r}\left(T^{n+k-1}\right)}{\mathrm{R}_{A}^{r}\left[(T+C)^{n}\right] \cap \mathrm{R}_{A}^{r}\left(T^{n+k-1}\right)} \leq \operatorname{dim} \mathrm{R}_{A}^{r}\left(C^{k}\right),
$$

and the proof is complete.

The following two theorems generalize [9, Theorem 2.2].

Theorem 4.3 Let $A \in \mathscr{L}(\mathrm{X})$ and $C, T \in \mathscr{L}_{\mathrm{N}(A)}(\mathrm{X})$. Suppose $\operatorname{dim} \mathrm{R}_{A}^{\ell}\left(C^{k}\right)<+\infty$ for some integer $k \geq 1$ and $C$ commutes with $T$. Then, $T$ has finite A-left ascent (resp., A-left descent) if and only if $T+C$ has finite A-left ascent (resp., A-left descent).

Proof Suppose that $\operatorname{asc}_{A}^{\ell}(T)=p<+\infty$. For $n \geq p$, let

$$
\begin{aligned}
& a_{n}=\operatorname{dim} \frac{\mathrm{N}_{A}^{\ell}\left(T^{n}\right)}{\mathrm{N}_{A}^{\ell}\left[(T+C)^{n+k-1}\right] \cap \mathbf{N}_{A}^{\ell}\left(T^{n}\right)}=\operatorname{dim} \frac{\mathbf{N}_{A}^{\ell}\left(T^{p}\right)}{\mathbf{N}_{A}^{\ell}\left[(T+C)^{n+k-1}\right] \cap \mathbf{N}_{A}^{\ell}\left(T^{p}\right)}, \\
& b_{n}=\operatorname{dim} \frac{\mathbf{N}_{A}^{\ell}\left[(T+C)^{n}\right]}{\mathbf{N}_{A}^{\ell}\left(T^{n+k-1}\right) \cap \mathbf{N}_{A}^{\ell}\left[(T+C)^{n}\right]}=\operatorname{dim} \frac{\mathbf{N}_{A}^{\ell}\left[(T+C)^{n}\right]}{\mathbf{N}_{A}^{\ell}\left(T^{p}\right) \cap \mathbf{N}_{A}^{\ell}\left[(T+C)^{n}\right]} .
\end{aligned}
$$

By Lemma 4.1, $a_{n} \leq \operatorname{dim} \mathrm{R}_{A}^{\ell}\left(C^{k}\right)<+\infty$. According to (1.3), the spaces $\left(\mathrm{N}_{A}^{\ell}\left[(T+C)^{i}\right]\right)_{i \in \mathbb{N}}$ are an increasing nest of subspaces, it follows that there is an integer $N \geq p$, such that $a_{n}=a_{N}$ for $n \geq N$. However, this implies

$$
\mathrm{N}_{A}^{\ell}\left[(T+C)^{i}\right] \cap \mathrm{N}_{A}^{\ell}\left(T^{p}\right)=\mathrm{N}_{A}^{\ell}\left[(T+C)^{N+k-1}\right] \cap \mathrm{N}_{A}^{\ell}\left(T^{p}\right), \quad \forall i \geq N+k-1 .
$$

On the other hand, we may interchange $T$ by $T+C$ and $C$ by $-C$ in Lemma 4.1 to conclude that $b_{n} \leq$ $\operatorname{dim} \mathrm{R}_{A}^{\ell}\left(C^{k}\right)<+\infty$. Since $b_{n}=\operatorname{dim}\left[\mathrm{N}_{A}^{\ell}\left[(T+C)^{n}\right]+\mathrm{N}_{A}^{\ell}\left(T^{p}\right)\right] / \mathrm{N}_{A}^{\ell}\left(T^{p}\right)$ (see [8, Lemma 2.2]), then $\left(b_{n}\right)_{n \in \mathbb{N}}$ is an increasing sequence, and so, there exists an integer $M \geq N+k-1$ such that $b_{n}=b_{M}$ for $n \geq M$. When combined with (4.1), this implies that $\mathrm{N}_{A}^{\ell}\left[(T+C)^{n}\right]=\mathrm{N}_{A}^{\ell}\left[(T+C)^{M}\right]$ for $n \geq M$, i.e., $\operatorname{asc}_{A}^{\ell}(T+C) \leq M<+\infty$.

For the converse implication, if $\operatorname{asc}_{A}^{\ell}(T+C)<+\infty$, then by the direct implication, $\operatorname{asc}_{A}^{\ell}(T)=\operatorname{asc}_{A}^{\ell}(T+$ $C-C)<+\infty$. proof.

The proof for the case when $T$ has finite, $A$-left descent is similar and will be omitted. This completes the 
Arguing as in the proof of [9, Theorem 2.2] or Theorem 4.3, with Lemma 4.2, we get the following theorem.

Theorem 4.4 Let $A \in \mathscr{L}(\mathrm{X})$ and $C, T \in \mathscr{L}_{\mathrm{R}(A)}(\mathrm{X})$. Suppose $\operatorname{dim} \mathrm{R}_{A}^{r}\left(C^{k}\right)<+\infty$ for some integer $k \geq 1$ and $C$ commutes with $T$. Then, $T$ has finite A-right ascent (resp., A-right descent) if and only if $T+C$ has finite A-right ascent (resp., A-right descent).

For $C, A \in \mathscr{L}(\mathrm{X})$ and $k \in \mathbb{N} \backslash\{0\}$, we have $\operatorname{dim} \mathrm{R}_{A}^{r}\left(C^{k}\right)<+\infty$ and $\operatorname{dim} \mathrm{R}_{A}^{\ell}\left(C^{k}\right)<+\infty$, when $\operatorname{dim} \mathrm{R}\left(C^{k}\right)<+\infty$. Then, as a consequence of Theorems 4.3 and 4.4, we obtain the following corollary.

Corollary 4.5 Let A, C, T $\in \mathscr{L}(\mathrm{X})$, such that $\operatorname{dim} \mathrm{R}\left(C^{k}\right)<+\infty$ for some integer $k \geq 1$ and $C$ commutes with $T$.

(1) If $C, T \in \mathscr{L}_{\mathrm{N}(A)}(\mathrm{X})$, then $T$ has finite A-left ascent (resp., A-left descent) if and only if $T+C$ has finite A-left ascent (resp., A-left descent).

(2) If $C, T \in \mathscr{L}_{\mathrm{R}(A)}(\mathrm{X})$, then $T$ has finite A-right ascent (resp., A-right descent) if and only if $T+C$ has finite A-right ascent (resp., A-right descent).

Now, we suppose that $\mathrm{X}$ is a Banach space, and let $\mathscr{B}(\mathrm{X})$ be the algebra of bounded linear operators on $\mathrm{X}$. The next lemma is used to show Lemma 4.7.

Lemma 4.6 Let $A, T \in \mathscr{B}(\mathrm{X})$, such that $T$ is compact. If $\mathrm{R}(A)$ is closed, then $\mathrm{R}_{A}^{r}\left[(\lambda I-T)^{n}\right]$ is closed for all $n \in \mathbb{N}$ and $\lambda \in \mathbb{C} \backslash\{0\}$.

Proof We begin with $n=1$. First, we see that $\mathrm{R}_{A}^{r}(\lambda I-T)=\mathrm{R}\left(S_{\lambda}\right)$, where $S_{\lambda}: \mathrm{R}(A) \longrightarrow \mathrm{X}$ is the operator given by $S_{\lambda}(x):=(\lambda I-T) x$. We now argue as in the proof of [19, Theorem 5.5-D], we get that $\mathrm{R}\left(S_{\lambda}\right)$ is closed, i.e., $\mathrm{R}_{A}^{r}(\lambda I-T)$ is closed.

For $n>1$, we write

$$
(\lambda I-T)^{n}=\lambda^{n}-T B,
$$

where $B$ is a certain bounded operator. Now, by [11, Theorem V.7.2], $K:=T B$ is a compact operator. The foregoing reasoning, applied to $\lambda^{n}-K$, shows that $\mathrm{R}_{A}^{r}\left[(\lambda I-T)^{n}\right]$ is closed. This completes the proof.

The next lemma is used to prove Theorems 4.8 and 4.9.

Lemma 4.7 Let $A \in \mathscr{B}(\mathrm{X})$ and $T \in \mathscr{B}(\mathrm{X})$ be compact.

(1) If $T(\mathrm{~N}(A)) \subset \mathrm{N}(A)$, then the A-left ascent of $\lambda I-T$ is finite, for all $\lambda \in \mathbb{C} \backslash\{0\}$.

(2) If $\mathrm{R}(A)$ is closed and $T(\mathrm{R}(A)) \subset \mathrm{R}(A)$, then the A-right descent of $\lambda I-T$ is finite, for all $\lambda \in \mathbb{C} \backslash\{0\}$.

Proof (1) We can prove that $A$-left ascent of $\lambda I-T$ is finite similarly as in the proof of [11, Theorem V.7.9]. Indeed, suppose $\operatorname{asc}_{A}^{\ell}(\lambda I-T)=+\infty$. Then, $\mathrm{N}_{A}^{\ell}\left[(\lambda I-T)^{n-1}\right]$ is a proper closed subset of $\mathrm{N}_{A}^{\ell}\left[(\lambda I-T)^{n}\right]$ for $n=1,2, \cdots$. By [11, Theorem II.3.5], there exists $x_{n} \in \mathrm{N}_{A}^{\ell}\left[(\lambda I-T)^{n}\right]$, such that $\left\|x_{n}\right\|=1$ and $\left\|x_{n}-x\right\| \geq \frac{1}{2}$ if $x \in \mathrm{N}_{A}^{\ell}\left[(\lambda I-T)^{n-1}\right]$. Assume $1 \leq m<n$ and let

$$
z=x_{m}+\lambda^{-1}(\lambda I-T) x_{n}-\lambda^{-1}(\lambda I-T) x_{m} .
$$

Then

$$
A(\lambda I-T)^{n-1} z=A(\lambda I-T)^{n-1} x_{m}+\lambda^{-1} A(\lambda I-T)^{n} x_{n}-\lambda^{-1} A(\lambda I-T)^{n} x_{m}=0,
$$

and so $z \in \mathrm{N}_{A}^{\ell}\left[(\lambda I-T)^{n-1}\right]$. Consequently $\left\|x_{n}-z\right\| \geq \frac{1}{2}$. However, we easily calculate that $T x_{n}-T x_{m}=$ $\lambda\left(x_{n}-z\right)$, and so, $\left\|T x_{n}-T x_{m}\right\| \geq \frac{\lambda}{2}>0$. This shows that $\left\{T x_{n}\right\}$ can have no convergent subsequence, in contradiction to the fact that $T$ is compact. Thus, $\operatorname{asc}_{A}^{\ell}(\lambda I-T)$ must be finite.

(2) The proof that $\operatorname{des}_{A}^{r}(\lambda I-T)$ is finite is similar. We first observe in Lemma 4.6 that $\mathrm{R}_{A}^{r}\left[(\lambda I-T)^{n}\right]$ is closed, for all $n \in \mathbb{N}$. If $\operatorname{des}_{A}^{r}(\lambda I-T)=+\infty, \mathrm{R}_{A}^{r}\left[(\lambda I-T)^{n+1}\right]$ would be a proper closed subset of $\mathrm{R}_{A}^{r}\left[(\lambda I-T)^{n}\right]$ for $n=0,1, \cdots$. We choose $y_{n} \in \mathrm{R}_{A}^{r}\left[(\lambda I-T)^{n}\right]$, so that $\left\|y_{n}\right\|=1$ and $\left\|y_{n}-y\right\| \geq \frac{1}{2}$ if $y \in \mathrm{R}_{A}^{r}\left[(\lambda I-T)^{n+1}\right]$. If $1 \leq m<n$ let

$$
w=y_{n}-\lambda^{-1}(\lambda I-T) y_{n}+\lambda^{-1}(\lambda I-T) y_{m} .
$$


We can write $y_{k}=(\lambda I-T)^{k} A x_{k}$ for some $x_{k} \in \mathrm{X}$. Thus

$$
w=(\lambda I-T)^{n} A x_{n}-\lambda^{-1}(\lambda I-T)^{n+1} A x_{n}+\lambda^{-1}(\lambda I-T)^{m+1} A x_{m},
$$

from which we see that $w \in \mathrm{R}_{A}^{r}\left[(\lambda I-T)^{m+1}\right]$. Therefore, $\left\|y_{m}-w\right\| \geq \frac{1}{2}$. However, $T y_{m}-T y_{n}=\lambda\left(y_{m}-w\right)$, and so, $\left\|T y_{m}-T y_{n}\right\| \geq \frac{\lambda}{2}$, and we obtain a contradiction, just as in the earlier argument. This completes the proof.

Recall that if $T$ and $C$ are bounded operators, such that $T$ is bijective and $C$ is compact which commutes with $T$, then the ascent and descent of $T+C$ are finite (see [9, Theorem 3.2]). We generalize this result to the $A$-left (resp., $A$-right) ascent and descent as in the following two theorems .

Theorem 4.8 Let $A, C, T \in \mathscr{B}(\mathrm{X})$, such that $\mathrm{N}(A)$ is an invariant subspace of $T$ and $C$. Suppose that $T$ is bijective and $C$ is a compact operator which commutes with $T$.

(1) The A-left descent of $T+C$ is finite.

(2) If $\operatorname{asc}_{A}^{\ell}(T)=0$, then the A-left ascent of $T+C$ is finite.

Proof (1) Let us first observe from Remark 1.6 and [9, Theorem 3.2] that

$$
\operatorname{des}_{A}^{\ell}(T+C) \leq \operatorname{des}(T+C)<+\infty .
$$

(2) Let $n$ be a non-negative integer. Since $(T+C)^{n}=T^{n}\left(I+C T^{-1}\right)^{n}$ and $\operatorname{asc}_{A}^{\ell}(T)=0$, we get

$$
\mathrm{N}_{A}^{\ell}\left[(T+C)^{n}\right]=\mathrm{N}_{A}^{\ell}\left[T^{n}\left(I+C T^{-1}\right)^{n}\right] \text { and } \mathrm{N}_{A}^{\ell}\left(T^{n}\right)=\mathrm{N}(A) .
$$

This shows that

$$
\begin{aligned}
x \in \mathrm{N}_{A}^{\ell}\left[(T+C)^{n}\right] & \Longleftrightarrow\left(I+C T^{-1}\right)^{n} x \in \mathrm{N}_{A}^{\ell}\left(T^{n}\right)=\mathrm{N}(A) \\
& \Longleftrightarrow x \in \mathrm{N}_{A}^{\ell}\left[\left(I+C T^{-1}\right)^{n}\right],
\end{aligned}
$$

and so, $\mathrm{N}_{A}^{\ell}\left[(T+C)^{n}\right]=\mathrm{N}_{A}^{\ell}\left[\left(I+C T^{-1}\right)^{n}\right]$. Now, since

$$
T^{-1}(\mathrm{~N}(A))=\{x \in \mathrm{X}: T x \in \mathrm{N}(A)\}=\left\{x \in \mathrm{X}: x \in \mathrm{N}_{A}^{\ell}(T)=\mathrm{N}(A)\right\}=\mathrm{N}(A),
$$

then $C T^{-1}(\mathrm{~N}(A)) \subset \mathrm{N}(A)$, and as $C T^{-1}$ is a compact operator (see [11, Theorem V.7.2]), by Lemma 4.7, it follows that $\operatorname{asc}_{A}^{\ell}(T+C)=\operatorname{asc}_{A}^{\ell}\left(I+C T^{-1}\right)<+\infty$. This completes the proof.

Theorem 4.9 Let $A, C, T \in \mathscr{B}(\mathrm{X})$, such that $\mathrm{R}(A)$ is an invariant subspace of $T$ and $C$. Suppose that $T$ is bijective and $C$ is a compact operator which commutes with $T$.

(1) The A-right ascent of $T+C$ is finite.

(2) If $\mathrm{R}(A)$ is closed and des ${ }_{A}^{r}(T)=0$, then the A-right descent of $T+C$ is finite.

Proof (1) Let us first observe from Remark 1.6 and [9, Theorem 3.2] that

$$
\operatorname{asc}_{A}^{r}(T+C) \leq \operatorname{asc}(T+C)<+\infty .
$$

(2) Let $n$ be a non-negative integer. Since $(T+C)^{n}=\left(I+C T^{-1}\right)^{n} T^{n}$ and $\operatorname{des}_{A}^{r}(T)=0$, we get

$$
\begin{aligned}
\mathrm{R}_{A}^{r}\left[(T+C)^{n}\right]=\mathrm{R}\left[\left(I+C T^{-1}\right)^{n} T^{n} A\right] & =\left(I+C T^{-1}\right)^{n}\left(\mathrm{R}_{A}^{r}\left(T^{n}\right)\right) \\
& =\left(I+C T^{-1}\right)^{n}(\mathrm{R}(A))=\mathrm{R}_{A}^{r}\left[\left(I+C T^{-1}\right)^{n}\right] .
\end{aligned}
$$

Now, since $T^{-1}(\mathrm{R}(A))=T^{-1}(\mathrm{R}(T A))=\mathrm{R}(A)+\mathrm{N}(T)=\mathrm{R}(A)$, then we infer that $C T^{-1}(\mathrm{R}(A)) \subset \mathrm{R}(A)$, and as $C T^{-1}$ is a compact operator, by Lemma 4.7, it follows that $\operatorname{des}_{A}^{r}(T+C)=\operatorname{des}_{A}^{r}\left(I+C T^{-1}\right)<+\infty$. This completes the proof. 


\section{Application to an $A$-adjoint of an operator on a Hilbert space}

This section is devoted to the study of the ascent and descent of an $A$-adjoint of an operator on a Hilbert space with an additional semi-inner product defined by a positive semidefinite operator $A$. We refer to $[2,3]$ for more information about the $A$-adjoint of an operator $T$.

Throughout $\mathrm{H}$ denotes a Hilbert space with inner product $\langle\cdot, \cdot\rangle$. For a given set $\mathrm{M} \subseteq \mathrm{H}$, the orthogonal subspace of $\mathrm{M}$ in $\mathrm{H}$ is denoted $\mathrm{M}^{\perp}$. For every $T \in \mathscr{B}(\mathrm{H})$, its adjoint is denoted by $T^{*}$. Given $A \in \mathscr{B}(\mathrm{H})$, such that $A^{*}=A$, then

$$
\mathrm{R}_{A}^{\ell}(T)^{\perp}=\mathrm{N}_{A}^{r}\left(T^{*}\right), \quad \mathrm{R}_{A}^{r}(T)^{\perp}=\mathrm{N}_{A}^{\ell}\left(T^{*}\right) .
$$

By $\mathscr{B}(\mathrm{H})^{+}$we denote the set of all positive (semidefinite) operators, i.e., $\mathscr{B}(\mathrm{H})^{+}=\{A \in \mathscr{B}(\mathrm{H}):\langle A x, x\rangle \geq$ $0, \forall x \in \mathrm{H}$ \}. Given $A \in \mathscr{B}(\mathrm{H})^{+}$, we consider the semi-inner product $\langle\cdot, \cdot\rangle_{A}$ defined by

$$
\langle x, y\rangle_{A}=\langle A x, y\rangle, x, y \in \mathrm{H} .
$$

Naturally, this semi-inner product induces a semi-norm, $\|\cdot\|_{A}$, defined by $\|x\|_{A}=\left\|A^{\frac{1}{2}} x\right\|$. Besides, this structure induces an adjoint operation. However, this operation is defined for no all bounded linear operator on $\mathrm{H}$, unless $A$ is bijective. Given $T \in \mathscr{B}(\mathrm{H})$, we say that $W \in \mathscr{B}(\mathrm{H})$ is an $A$-adjoint of $T$ if

$$
\langle T x, y\rangle_{A}=\langle x, W y\rangle_{A}, \quad \forall x, y \in \mathrm{H}
$$

or, equivalently, if $A W=T^{*} A$. Since the equation $A X=T^{*} A$ is not solvable for every $T \in \mathscr{B}(\mathrm{H})$, then not every $T \in \mathscr{B}(\mathrm{H})$ admits an A-adjoint operator. Thus, we shall denote by

$$
\mathscr{B}_{A}(\mathrm{H}):=\{T \in \mathscr{B}(\mathrm{H}): T \text { admits } A \text {-adjoint }\} \text {. }
$$

In particular, given $T \in \mathscr{B}_{A}(\mathrm{H})$ and $\mathrm{M}$ a closed subspace of $\mathrm{H}$, such that $\mathrm{M}+\mathrm{N}(A)=\mathrm{H}$, we shall denote by $T T^{\sharp}$ the reduced solution for $\mathrm{M}$ of

$$
A X=T^{*} A .
$$

This means that $A T^{\sharp \mathrm{M}}=T^{*} A$ and $\mathrm{R}\left(T^{\sharp \mathrm{M}}\right) \subset \mathrm{M}$. In such case, we shall say that $T^{\sharp \mathrm{M}}$ is a reduced A-adjoint of $T$. In addition, when no confusion arises, we shall simply write $T^{\sharp}$ instead of $T^{\sharp}$. Note that fixed the subspace $M$, there exists a unique solution of (5.1) with range included in M. Moreover, it is easy to prove that

$$
\mathrm{N}\left(T^{\sharp}\right)=\mathrm{N}_{A}^{\ell}\left(T^{\sharp}\right)=\mathrm{N}_{A}^{r}\left(T^{*}\right), \quad \mathrm{R}_{A}^{r}\left(T^{*}\right)=\mathrm{R}_{A}^{\ell}\left(T^{\sharp}\right) .
$$

In addition, observe that if $T^{*} A=A T$ then it does not mean, in general, that $T=T^{\sharp}$. In fact, $T=T^{\sharp}$ if and only if $T^{*} A=A T$ and $\mathrm{R}(T) \subseteq \mathrm{M}$.

If $T \in \mathscr{B}_{A}(\mathrm{H})$, from [3, Lemma 1.1], for all $n \in \mathbb{N}$, we see that $T^{n} \in \mathscr{B}_{A}(\mathrm{H})$ and

$$
\left(T^{\sharp}\right)^{n}=\left(T^{n}\right)^{\sharp} .
$$

Now, by combining (5.2) and (5.3), it follows that:

$$
\mathrm{N}\left(T^{\sharp n}\right)=\mathrm{N}_{A}^{\ell}\left(T^{\sharp n}\right)=\mathrm{N}_{A}^{r}\left(T^{* n}\right), \quad \mathrm{R}_{A}^{r}\left(T^{* n}\right)=\mathrm{R}_{A}^{\ell}\left(T^{\sharp n}\right),
$$

for all $n \in \mathbb{N}$. From [4], we have $\mathscr{B}_{A}(\mathrm{H})=\left\{T \in \mathscr{B}(\mathrm{H}): \mathrm{R}\left(T^{*} A\right) \subseteq \mathrm{R}(A)\right\}$ i.e., $T \in \mathscr{B}_{A}(\mathrm{H})$ if and only if $\mathrm{R}(A)$ is an invariant subspace of $T^{*}$. Since $A=A^{*}$, then $\mathrm{N}(A)=\mathrm{R}(A)^{\perp}$, and by [10, Problem 4.3], we obtain that $\mathrm{N}(A)$ is an invariant subspace of $T$ when $T \in \mathscr{B}_{A}(\mathrm{H})$. In addition, if $\mathrm{R}(A)$ is closed then $T \in \mathscr{B}_{A}(\mathrm{H})$ if and only if $\mathrm{N}(A)$ is an invariant subspace of $T$. Hence, by (5.4), it follows that:

$$
\operatorname{asc}\left(T^{\sharp}\right)=\operatorname{asc}_{A}^{r}\left(T^{*}\right)=\operatorname{asc}_{A}^{\ell}\left(T^{\sharp}\right),
$$

for all $A \in \mathscr{B}(\mathrm{H})^{+}$and $T \in \mathscr{B}_{A}(\mathrm{H})$. On the other hand, we see by Lemma 2.3 and (5.4) that

$$
\frac{\mathrm{R}_{A}^{\ell}\left(T^{\sharp n}\right)}{\mathrm{R}_{A}^{\ell}\left(T^{\sharp^{n+1}}\right)} \cong \frac{\mathrm{H}}{\mathrm{R}\left(T^{\sharp}\right)+\mathrm{N}_{A}^{\ell}\left(T^{\sharp^{n}}\right)}=\frac{\mathrm{H}}{\mathrm{R}\left(T^{\sharp}\right)+\mathrm{N}\left(T^{\sharp^{n}}\right)} \cong \frac{\mathrm{R}\left(T^{\sharp n}\right)}{\mathrm{R}\left(T^{\sharp n+1}\right)},
$$


for all $n \in \mathbb{N}$. A combination of (5.4) and (5.6) leads to

$$
\operatorname{des}\left(T^{\sharp}\right)=\operatorname{des}_{A}^{\ell}\left(T^{\sharp}\right)=\operatorname{des}_{A}^{r}\left(T^{*}\right),
$$

for all $A \in \mathscr{B}(\mathrm{H})^{+}$and $T \in \mathscr{B}_{A}(\mathrm{H})$. Consequently

$$
\operatorname{des}(T)=\operatorname{des}_{A}^{\ell}(T) \text { and } \operatorname{asc}(T)=\operatorname{asc}_{A}^{\ell}(T)
$$

if $T^{\sharp}=T$.

Let $\mathrm{X}$ be a linear space, for $A, T \in \mathscr{L}(\mathrm{X})$ and $n \in \mathbb{N}$, we define the following quantities :

$$
\begin{gathered}
A-\alpha_{n}^{\ell}(T)=\operatorname{dim} \mathrm{N}_{A}^{\ell}(T) \cap \mathrm{R}_{A}^{\ell}\left(T^{n}\right), \quad A-\alpha_{n}^{r}(T)=\operatorname{dim} \mathrm{N}_{A}^{r}(T) \cap \mathrm{R}_{A}^{r}\left(T^{n}\right), \\
A-\beta_{n}^{\ell}(T)=\operatorname{dim} \frac{\mathrm{X}}{\mathrm{R}_{A}^{\ell}(T)+\mathrm{N}_{A}^{\ell}\left(T^{n}\right)}, \quad A-\beta_{n}^{r}(T)=\operatorname{dim} \frac{\mathrm{X}}{\mathrm{R}_{A}^{r}(T)+\mathrm{N}_{A}^{r}\left(T^{n}\right)} .
\end{gathered}
$$

Lemma 1.2 shows that $\left(A-\alpha_{n}^{\ell}(T)\right)_{n \geq 0}$ and $\left(A-\beta_{n}^{r}(T)\right)_{n \geq 0}$ are decreasing sequences. By the same lemma, $\left(A-\alpha_{n}^{r}(T)\right)_{n \geq 0}\left(\right.$ resp., $\left.\left(A-\beta_{n}^{\ell}(T)\right)_{n \geq 0}\right)$ is a decreasing sequence when $T \in \mathscr{L}_{\mathrm{R}(A)}(\mathrm{X})\left(\right.$ resp., $\left.T \in \mathscr{L}_{\mathrm{N}(A)}(\mathrm{X})\right)$. This leads to the introduction of the following new concepts :

- The $A$-left $g$-ascent, $g$-asc ${ }_{A}^{\ell}(T)$, of $T \in \mathscr{L}(\mathrm{X})$ is defined by

$$
g-\operatorname{asc}_{A}^{\ell}(T)=\inf \left\{n \in \mathbb{N}: A-\alpha_{n}^{\ell}(T)=0\right\}
$$

- The $A$-left $g$-descent, $g$ - $\operatorname{des}_{A}^{\ell}(T)$, of $T \in \mathscr{L}_{\mathrm{N}(A)}(\mathrm{X})$ is defined by

$$
g-\operatorname{des}_{A}^{\ell}(T)=\inf \left\{n \in \mathbb{N}: A-\beta_{n}^{\ell}(T)=0\right\}
$$

- The $A$-right $g$-ascent, $g$-asc ${ }_{A}^{r}(T)$, of $T \in \mathscr{L}_{\mathrm{R}(A)}(\mathrm{X})$ is defined by

$$
g-\operatorname{asc}_{A}^{r}(T)=\inf \left\{n \in \mathbb{N}: A-\alpha_{n}^{r}(T)=0\right\} .
$$

- The $A$-right $g$-descent, $g$ - $\operatorname{des}_{A}^{r}(T)$, of $T \in \mathscr{L}(\mathrm{X})$ is defined by

$$
g-\operatorname{des}_{A}^{r}(T)=\inf \left\{n \in \mathbb{N}: A-\beta_{n}^{r}(T)=0\right\},
$$

where as usual the infimum over the empty set is taken to be $+\infty$. It follows from [8, Lemmas 3.1 and 3.2] that the case when $A=I$ represents the ascent and descent of $T$.

Finally, we set some open problems for the generalization of ascent and descent of a linear operator in a linear space.

Question 5.1 Can some results known for the ascent and descent of a linear operator be extended to the new concepts $A$-left (resp., $A$-right) $g$-ascent and $g$-descent?

Open Access This article is licensed under a Creative Commons Attribution 4.0 International License, which permits use, sharing, adaptation, distribution and reproduction in any medium or format, as long as you give appropriate credit to the original author(s) and the source, provide a link to the Creative Commons licence, and indicate if changes were made. The images or other third party material in this article are included in the article's Creative Commons licence, unless indicated otherwise in a credit line to the material. If material is not included in the article's Creative Commons licence and your intended use is not permitted by statutory regulation or exceeds the permitted use, you will need to obtain permission directly from the copyright holder. To view a copy of this licence, visit http://creativecommons.org/licenses/by/4.0/. 


\section{References}

1. Andruchow, E.; Corach, G.; Mbekhta, M.: Split Partial Isometries. Complex Anal. Oper. Theory. 7, 813-829 (2013)

2. Arias, M.L.; Corach, G.; Gonzalez, M.C.: Partial isometries in semi-Hilbertian spaces. Linear Algebra Appl. 428, 1460-1475 (2008)

3. Arias, M.L.; Mbekhta, M.: A-partial isometries and generalized inverses. Linear Algebra Appl. 439, 1286-1293 (2013)

4. Douglas, R.G.: On majorization, factorization and range inclusion of operators in Hilbert space. Proc. Amer. Math. Soc. 17, 413-416 (1966)

5. Fongi, G.; Gonzaleza, M.C.: Partial isometries and pseudoinverses insemi-Hilbertian spaces. Linear Algebra Appl. 495, 324-343 (2016)

6. Z. Garbouj, H. Skhiri, Semi-generalized partial isometries. Results Math. 75, 15 (2020)

7. H. Heuser, Über Operatoren mit endlichen Defekten. Inaugural-Dissertation, Universitüt Tübingen (1956)

8. M. A. Kaashoek, Ascent, descent, nullity and defect, a note on a paper by A. E. Taylor. Math. Ann. 172, 105-115 (1967)

9. Kaashoek, M.A.; Lay, D.C.: Ascent, descent, and commuting perturbations. Trans. Amer. Math. Soc. 169, 35-47 (1972)

10. Carlos, S.: Kubrusly, Hilbert space operators: a problem solving approach. Birkhäuser, Boston (2003)

11. Lay, D.C.; Taylor, A.E.: Introduction to Functional Analysis, 2nd edn. John Wiley \& Sons, New York (1980)

12. Riesz, F.: Uber lineare Functionalgleichungen. Acta Math. 41, 71-98 (1918)

13. Salhi, A.; Zerouali, E.H.: Decomposition of partial isometries with finite ascent. Adv. Oper. Theory. 5, 64-82 (2020)

14. Sandovici, A.; de Snoo, H.; Winkler, H.: Ascent, descent, nullity, defect, and related notions for linear relations in linear spaces. Linear Algebra Appl. 423, 456-497 (2007)

15. Sid Ahmed, O.A.M.; Saddi, A.: A-m-isometric operators in semi-Hilbertian spaces. Linear Algebra Appl. 436, 3930-3942 (2012)

16. Suciu, L.: Quasi-isometries in semi-Hilbertian spaces. Linear Algebra Appl. 430, 2474-2487 (2009)

17. Suciu, L.: Some invariant subspaces for A-contractions and applications. Extracta Math. 21(3), 221-247 (2006)

18. Taylor, A.E.: Theorems on Ascent, Descent, Nullity and Defect of Linear Operators. Math. Ann. 163, 18-49 (1966)

19. Taylor, A.E.: Introduction to Functional Analysis. John Wiley \& Sons, New York (1958)

Publisher's Note Springer Nature remains neutral with regard to jurisdictional claims in published maps and institutional affiliations. 